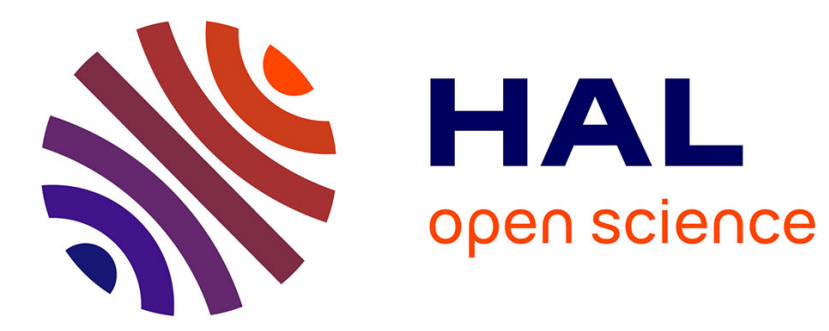

\title{
Assume-guarantee contracts for continuous-time systems
}

Adnane Saoud, Antoine Girard, Laurent Fribourg

\section{To cite this version:}

Adnane Saoud, Antoine Girard, Laurent Fribourg. Assume-guarantee contracts for continuous-time systems. Automatica, 2021, 134, 10.1016/j.automatica.2021.109910 . hal-02196511v3

\section{HAL Id: hal-02196511 \\ https://hal.science/hal-02196511v3}

Submitted on 24 Jun 2021

HAL is a multi-disciplinary open access archive for the deposit and dissemination of scientific research documents, whether they are published or not. The documents may come from teaching and research institutions in France or abroad, or from public or private research centers.
L'archive ouverte pluridisciplinaire HAL, est destinée au dépôt et à la diffusion de documents scientifiques de niveau recherche, publiés ou non, émanant des établissements d'enseignement et de recherche français ou étrangers, des laboratoires publics ou privés. 


\title{
Assume-guarantee contracts for continuous-time systems *
}

\author{
Adnane Saoud ${ }^{\mathrm{a}, \mathrm{b}}$ Antoine Girard ${ }^{\mathrm{a}}$ Laurent Fribourg ${ }^{\mathrm{b}}$ \\ ${ }^{a}$ Université Paris-Saclay, CNRS, CentraleSupélec \\ Laboratoire des Signaux et Systèmes \\ 91190, Gif-sur-Yvette, France \\ ${ }^{\mathrm{b}}$ Université Paris-Saclay, CNRS, ENS Paris-Saclay \\ Laboratoire de Méthodes Formelles \\ 91190, Gif-sur-Yvette, France
}

\begin{abstract}
Many techniques for verifying properties of continuous-time systems are limited to systems of moderate size. In this paper, we propose an approach based on assume-guarantee contracts and compositional reasoning for verifying properties of a broad class of continuous-time systems consisting of interconnected components. The notion of assume-guarantee contracts makes it possible to divide responsibilities among the system components: a contract specifies the property that a component must fulfill under some assumptions on the behavior of its environment (i.e. of the other components). We define weak and strong semantics of assume-guarantee contracts.c We then establish a certain number of results for compositional reasoning, which allow us to show that a global assume-guarantee contract of the whole system is satisfied when all components satisfy their own contracts. We show that the weak satisfaction of the contract is sufficient to deal with interconnections described by a directed acyclic graph, while strong satisfaction is needed to reason about general interconnections containing cycles. Specific results for systems described by differential inclusions and invariance assume-guarantee contracts are then developed. Finally, we show how the proposed assume-guarantee framework can recast different versions of the small-gain theorem as a particular case. Throughout the paper, the main results are illustrated using simple examples.
\end{abstract}

Key words: component-based design, assume-guarantee contracts, prefix-closed properties, small-gain theorem.

\section{Introduction}

Cyber-physical systems (CPS) result from integrations of computational devices with physical processes and are to become ubiquitous in modern societies (autonomous vehicles, smart buildings, robots, etc.) (see [25] and the references therein). Despite considerable progress in the field, current techniques apply to system of moderate complexity (the complexity is quantified by the number of interacting components). Thus, the design of complex

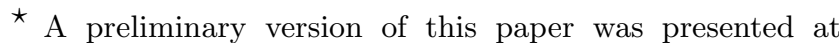
the 16th European Control Conference. This research was supported by Labex DigiCosme (project ANR-11-LABEX0045-DIGICOSME) operated by ANR as part of the program "Investissement d'Avenir" Idex Paris Saclay (ANR11-IDEX-0003-02). This project was partially supported by the European Research Council (ERC) under the European Union's Horizon 2020 research and innovation programme (grant agreement No 725144). Corresponding author A. Saoud; e-mail: Adnane.Saoud@12s.centralesupelec.fr, Antoine.Girard@12s.centralesupelec.fr, fribourg@lsv.enscachan.fr
}

CPS requires to divide large design problems in smaller sub-problems that can be solved using existing tools.

Compositional approaches for the analysis and the design of dynamical systems have been long known in the field of control theory, where the celebrated small-gain theorem $[12,19,21]$ makes it possible to prove stability of a system from the stability of its components. Other compositional approaches for the analysis and design of cyber-physical systems have been mainly initiated in the field of computer science $[1,15,18]$.

The study of properties of dynamical systems using decentralized approaches has been an ongoing research area in recent years $[3,8,9,13,29,30,37]$. Other compositional approaches, using formal methods and symbolic techniques, are presented in $[10,22,24,26-$ $28,32,35,38,40]$. All these works develop efficient computational techniques by making specific assumptions on the classes of dynamical systems and of properties to which they can be applied. 
In the current work, we aim at proposing a general theoretical framework and thus we make weak assumptions on systems and properties. We initiate a highlevel framework for verifying properties of complex systems, consisting of interconnected components, using a contract-based approach [6]. Each component is assigned an assume-guarantee contract, which specifies the property that the component must fulfill under assumption about its environment (i.e. the other components). We introduce contracts and define weak and strong semantics. We then establish results that allow us to reason compositionally using assume-guarantee contracts: i.e. if all components satisfy their own contract then a global contract of the whole system is satisfied. We show that the weak satisfaction of the contract is sufficient to deal with interconnections described by a directed acyclic graph, while strong satisfaction is needed to reason about general interconnections containing cycles. We then show that for systems described by differential inclusions and invariance assume-guarantee contracts, weak satisfaction of contracts is sufficient to reason on general interconnections. Finally, we show how the proposed assume-guarantee framework can recast different versions of the small-gain theorem as a particular case.

There are several advantages in using contract based design for CPS. Firstly, by dividing a complex design problem into several smaller sub-problems, one is able to address design challenges that would be out of reach of current state-of-the-art design methods. Secondly, contractbased design makes it possible to replace a component without jeopardizing the behavior of the overall system: one just has to make sure that the new component satisfies the assigned contract. Thirdly, components are reusable when similar contracts appear in the decomposition of a global contract.

Let us emphasize that the main objective of the paper is to provide compositionality rules on how to go from the satisfaction of local contracts for the components to the satisfaction of a global contract for the whole interconnected system. The question on how to construct the local contracts from the global one is not investigated in this paper, but have been explored in other works in the literature for continuous-time in [41] and [6, Section $8.6]$, and for discrete-time $[7,14,16]$ systems.

The present paper focuses on the theoretical development of a general framework for contract-based reasoning. Applications of this framework to the design of symbolic controllers are reported in $[32,33,41]$.

Related work: Contract based design for CPS have been widely studied in [6]. Our approach differs from the one proposed in [6] in several directions:

- In [6] the contracts need to be checked for the whole time domain on which trajectories are defined. In this paper, the satisfaction of contracts is defined in a progressive and time-dependant way, which makes it possible to detect the instant at which the contract is violated.

- While in [6] assumptions and guarantees are defined over all the set of variables, namely inputs and outputs, in this paper, we focus on the case where assumptions are defined on the inputs and guarantees on the states and outputs. Indeed, this restriction allows us to provide simple compositionality results.

- The use of saturated contracts is crucial in applying the contract framework proposed in [6], which requires being able to compute unions and complements of different assertions. In this paper, saturation of the contracts is not required, and one can deal directly with the contracts as they are.

Assume-guarantee reasoning has been previously used in control theory. The authors in [20] presented a compositionality result for linear dynamical systems based on the notion of simulation introduced in [39]. In spirit, our work is closer to the framework presented in [23] for verifying general properties using parametric assumeguarantee contracts and compositional reasoning by means of small-gain theorems. Our approach differs from the one proposed in [23] in the following directions:

- The approach proposed in [23] follows the framework of [6], which makes a noticeable difference with our results;

- In [23], the results hold only for discrete-time systems, while our approach makes it possible to deal with both continuous and discrete-time systems.

- The main compositionality result in [23] requires to assume that at least one component satisfies a contract (for some parameter value), independently of the behavior of other components. This breaks the circularity of implications of the assume-guarantee contracts, which is arguably the main difficulty in contract-based design, and the reason why we introduce weak and strong semantics for assume-guarantee contracts, which has not been done in [23].

- In [23], the authors show how their framework makes it possible to reprove the classical bounded input bounded output small-gain theorem for discretetime systems. In our paper we show how the proposed framework allows to recast the bounded input bounded output small gain theorem for continoustime systems. Moreover, we provide a new small-gain result for the notion of growth bound, which has not been proposed before in the control literature.

The paper is organized as follows. In Section 2, we introduce the class of prefix-closed properties. In Section 3, we introduce the class of systems and interconnections considered throughout the paper. In Section 4, we introduce assume-guarantee contracts, their weak and strong semantics. We then establish compositionality results for reasoning about interconnected systems in Section 5 . 
In Section 6, we develop specific results for systems described by differential inclusions and invariance assumeguarantee contracts. Finally, in Section 7, we show that different versions of the classical small-gain theorem can be recast as particular applications of our framework. Throughout the paper, simple examples are used as illustrations of the main results.

Due to space constraints, the results for discrete-time systems and contracts are omitted and can be found in [36, Chapter 2]. A preliminary version of this work has been presented in the conference paper [34]. The current paper extends the approach in different directions. First, we generalize the approach from cascade and feedback compositions to any composition structure and from invariance to more general properties. Second, while in [34], we have shown that for systems described by Lipschitz differential inclusions, the notion of invariance relative to assume-guarantee contracts implies the weak satisfaction. In the current work, we show the converse result. Finally, in the current work, we show that different versions of the classical small-gain theorem can be recovered using our framework.

Notation: $\mathbb{R}, \mathbb{R}_{0}^{+}, \mathbb{R}^{+}$and $\mathbb{N}$ denote the set or reals, nonnegative reals, positive reals and positive integers respectively. The set of continuous-time domains is $\mathbb{E}\left(\mathbb{R}_{0}^{+}\right)=$ $\left\{[0, a], a \in \mathbb{R}_{0}^{+}\right\} \cup\left\{[0, a), a \in \mathbb{R}^{+}\right\} \cup\left\{\mathbb{R}_{0}^{+}\right\}$. For $Z \subseteq \mathbb{R}^{n}$, we denote by $M(Z)$ the set of continuous-time maps $z: E \rightarrow Z$, where $E \in \mathbb{E}\left(\mathbb{R}_{0}^{+}\right)$. For $q \in \mathbb{N}_{0}^{+}, \mathcal{D}^{q}$ denotes the set of continuous-time maps q times differentiable. For $x \in \mathbb{R}^{n},\|x\|$ denotes the infinity norm. For a continuous-time map $x: E \rightarrow \mathbb{R}^{n}$ and for $t \in E$, $\left\|x_{\mid[0, t]}\right\|_{\infty}$ denotes the essential supremum of $x: E \rightarrow$ $\mathbb{R}^{n}$ on $[0, t]$. Given a space $X$ equipped with a metric $d: X \times X \rightarrow \mathbb{R}_{0}^{+}$, the closure of the set $A \subseteq X$ is denoted $\operatorname{cl}(A)$ and its complement is denoted $\bar{A}$. For $\varepsilon>0$ and $x \in X$, the ball with center in $x$ and a radius $\varepsilon$ is $\mathcal{R}_{\varepsilon}(x)=\{y \in X \mid d(x, y) \leq \varepsilon\}$. A continuous function $\gamma: \mathbb{R}_{0}^{+} \rightarrow \mathbb{R}_{0}^{+}$is said to belong to class $\mathcal{K}$ if it is strictly increasing and $\gamma(0)=0 ; \gamma$ is said to belong to class $\mathcal{K}_{\infty}$ if $\gamma$ is $\mathcal{K}$ and $\gamma(r) \rightarrow \infty$ as $r \rightarrow \infty$. A continuous function $\beta: \mathbb{R}_{0}^{+} \times \mathbb{R}_{0}^{+} \rightarrow \mathbb{R}_{0}^{+}$is said to belong to class $\mathcal{K} \mathcal{L}$ if, for each fixed $s$, the map $\beta(\cdot, s)$ belongs to class $\mathcal{K}$, and for each fixed nonzero $r$, the map $\beta(r, \cdot)$ is strictly decreasing and $\beta(r, s) \rightarrow 0$ as $s \rightarrow \infty$. Given a set $X, 2^{X}$ is used to denote its power set.

\section{Preliminaries on prefix-closed sets}

Given a set $Z \subseteq \mathbb{R}^{n}$, prefix closed sets are subsets $P \subseteq$ $M(Z)$ that can be defined as follows: if a trajectory $z$ : $E \rightarrow Z$ belongs to the prefix-closed set $P$, then any prefix of $z$ belongs to $P$. In this part, we first give a formal definition of a prefix-closed set, we then give a necessary and sufficient condition for a set to be prefixclosed, finally we give some examples of such sets.
Definition 1 Let $Z \subseteq \mathbb{R}^{n}$. Let $z: E \rightarrow Z$ and $z^{\prime}: E^{\prime} \rightarrow$ $Z$ in $M(Z)$. $z$ is said to be a prefix of $z^{\prime}$ and denoted $z \in \operatorname{pref}\left(z^{\prime}\right)$ if $E \subseteq E^{\prime}$ and for all $t \in E, z(t)=z^{\prime}(t)$. In this case, $z$ can be seen as a restriction of $z^{\prime}$ and is also denoted $z=z_{\mid E}^{\prime}$.

This notion is generalized toward sets of continuoustime maps in the usual way: for $A \subseteq M(Z), \operatorname{pref}(A)=$ $\bigcup_{z \in A} \operatorname{pref}(z)$.

Definition 2 Let $Z \subseteq \mathbb{R}^{n}$ and $P \subseteq M(Z) . P$ is said to be prefix-closed if the following logical implication is satisfied: $z \in P$ and $\hat{z} \in \operatorname{pref}(z) \Rightarrow \hat{z} \in P$.

In the following we will give a characterization of prefixclosed sets.

Proposition 1 Let $Z \subseteq \mathbb{R}^{n}$ and $P \subseteq M(Z)$. $P$ is prefixclosed if and only if $\operatorname{pref}(P)=P$.

PROOF. Suppose that $\operatorname{pref}(P)=P$ and let us prove that $P$ is prefix-closed. Let $z \in P$ and $\hat{z} \in \operatorname{pref}(z)$. Since $z \in P$, we have $\hat{z} \in \operatorname{pref}(z) \subseteq \operatorname{pref}(P)=P$. Then, $\hat{z} \in P$ and $P$ is prefix-closed. Now suppose that $P$ is prefix-closed and let us prove that $\operatorname{pref}(P)=P$. The inclusion $P \subseteq \operatorname{pref}(P)$ is verified by definition of the prefix. Let $\hat{z} \in \operatorname{pref}(P)$, then there exists $z \in P$ such that $\hat{z} \in \operatorname{pref}(z)$. Since $P$ is prefix-closed we get $\hat{z} \in P$. Then, $\operatorname{pref}(P) \subseteq P$ which ends the proof.

It can be seen that our notion of prefix-closed sets is relatively close to the notion of safety for linear time properties in [5]. Indeed, the only difference is that we are considering finite and infinite trajectories, while the linear time properties are defined in [5] over infinite words. In the following we give some examples of prefix-closed sets. This notion allows us to represent different type of properties such as invariance or systems described by differential inclusions.

Example 1 (Invariance) Let the set $S \subseteq \mathbb{R}^{n}$ such that $S \neq \emptyset$ and let us define:

$$
A=\left\{z: E \rightarrow \mathbb{R}^{n} \in M\left(\mathbb{R}^{n}\right) \mid \forall t \in E, z(t) \in S\right\} .
$$

The set A represents the set of continuous-time trajectories that belongs to the set $S$ for all time.

Example 2 Let $S_{1}, S_{2} \ldots, S_{q} \subseteq \mathbb{R}^{n}$ such that for all $i \in\{1,2, \ldots, q\}, S_{i} \neq \emptyset$ and let us define:

$$
\begin{array}{r}
A=\left\{z: E \rightarrow \mathbb{R}^{n} \in M\left(\mathbb{R}^{n}\right) \cap \mathcal{D}^{q} \mid \forall i \in\{1,2, \ldots, q\},\right. \\
\left.\forall t \in E, z^{(i)}(t) \in S_{i}\right\}
\end{array}
$$


Where $z^{(i)}$ denotes the ith derivative of $z$. The set $A$ in this example represents the set of continuous-time trajectories for which the $i$-th time derivative, $i \in\{1,2, \ldots, q\}$, belongs to the set $S_{i}$ for all time.

Example 3 (Differential inclusion) Let $F: \mathbb{R}^{n} \rightarrow 2^{\mathbb{R}^{n}}$ be a set-valued map we define:

$A=\left\{z: E \rightarrow \mathbb{R}^{n} \in M\left(\mathbb{R}^{n}\right) \cap \mathcal{D}^{1} \mid \forall t \in E, \dot{z}(t) \in F(z(t))\right\}$

An example of a non prefix-closed property is the reachability property described as follows:

Example 4 Let the set $K \subseteq \mathbb{R}^{n}$ such that $K \neq \emptyset$ and let us define:

$$
A=\left\{z: E \rightarrow \mathbb{R}^{n} \in M\left(\mathbb{R}^{n}\right) \mid \exists t \in E, z(t) \in K\right\} .
$$

\section{$3 \quad$ Systems and interconnections}

\subsection{Systems}

In this section, we introduce the classes of systems and interconnections considered throughout this paper, it is important to note that the classes of systems used in the paper are quite general, and includes deterministic and nondeterministic systems, described by difference or differential equations and inclusions and allows us to deal with phenomena such as sampling, time delays.

Definition 3 A continuous-time system is a tuple $\Sigma=$ $\left(W_{1}, W_{2}, X, Y, \mathcal{T}\right)$ where

- $W_{1} \subseteq \mathbb{R}^{m_{1}}, W_{2} \subseteq \mathbb{R}^{m_{2}}, X \subseteq \mathbb{R}^{n}$ and $Y \subseteq \mathbb{R}^{p}$, are the sets in which external and internal inputs, states, and outputs take their values;

- $\mathcal{T} \subseteq M\left(W_{1} \times W_{2} \times X \times Y\right)$ is a set of continuous-time trajectories.

\subsection{Interconnections}

We first introduce some notations for interconnected systems. A network of systems consists of a collection of $N \in \mathbb{N}_{>0}$ systems $\left\{\Sigma^{1}, \ldots, \Sigma^{N}\right\}$, a set of vertices $I=$ $\{1, \ldots, N\}$ and a binary connectivity relation $\mathcal{I} \subseteq I \times I$ where each vertex $i \in I$ is labelled with the system $\Sigma^{i}$. For $i \in I$, we define $\mathcal{N}(i)=\{j \in I \mid(j, i) \in \mathcal{I}\}$ as the set of neighbouring components from which the incoming edges originate. We define $I_{\text {init }}=\{i \in I \mid \mathcal{N}(i)=\emptyset\}$ as the set of components for which there exist no incoming edge.

Given a directed graph $\mathcal{G}=(I, \mathcal{I})$ over the set of vertices $I=\{1, \ldots, N\}$ and binary connectivity relation $\mathcal{I}$. A walk is a sequence $\sigma=a_{1} a_{2} \ldots a_{m}$ such that for all $i \in\{1, \ldots, m-1\},\left(a_{i}, a_{i+1}\right) \in \mathcal{I}$, in such case we say that $a_{i}$ is an element of $\sigma$. For a walk $\sigma$, if the vertices $a_{1}, \ldots, a_{m-1}$ are distinct and $a_{1}=a_{m}$, then $\sigma$ is a cycle. The set of all cycles is denoted $\zeta=\left\{\zeta_{1}, \ldots, \zeta_{p}\right\}, p \in \mathbb{N}$.

Remark 1 We recall that for a directed graph, if we drop one edge of every cycle in the graph, a directed acyclic graph (DAG) denoted $\mathcal{G}^{D A G}$ is obtained. In such case, the set of initial vertices for the new directed acyclic graph is characterized by $I_{\text {init }}^{D A G} \subseteq I_{\text {init }} \cup A$, where $A \subseteq I$ is the set of vertices to which we dropped an edge. An illustration of this approach is given in Figure 1.
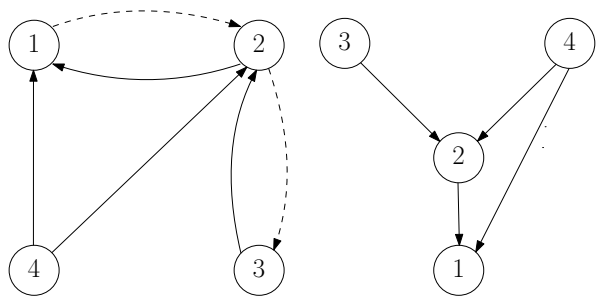

Fig. 1. Left: A graph $\mathcal{G}$ of four vertices, containing two cycles. The set of initial vertices is $I_{\text {init }}=\{4\}$. Right: A new DAG graph is constructed by removing dashed edges $\mathcal{G}^{\mathrm{DAG}}$. The set of initial vertices is $I_{\text {init }}^{\mathrm{DAG}}=\{4,3\} \subseteq I_{\text {init }} \cup A=\{4\} \cup\{2,3\}$.

In the following, we consider interconnections of continuous-time systems defined as follows:

Definition 4 Let $\left\{\Sigma^{i}\right\}_{i \in I}$ be a collection of systems and $\mathcal{I} \subseteq I \times I$ a binary connectivity relation. We say that $\left\{\Sigma^{i}\right\}_{i \in I}$ is compatible for composition with respect to $\mathcal{I}$, if for each $i \in I$, we have $\prod_{j \in \mathcal{N}(i)} Y^{j} \subseteq W_{2}^{i}$, i.e., the internal input space of $\Sigma^{i}$ is a superset of the Cartesian product of the output spaces of all the neighbors in $\mathcal{N}(i)$. The composed system $\Gamma$ denoted $\left\langle\left(\Sigma^{i}\right)_{i \in I}, \mathcal{I}\right\rangle$, is given by a tuple $\Gamma=\left(W_{1},\{0\}, X, Y, \mathcal{T}\right)$ where:

- $W_{1}=\prod_{i \in I} W_{1}^{i}$;

- $X=\prod_{i \in I} X^{i}$

- $Y=\prod_{i \in I} Y^{i}$;

- $\left(w_{1}, 0, x, y\right): E \rightarrow W_{1} \times\{0\} \times X \times Y \in \mathcal{T}$ is a trajectory of $\Gamma$ if and only if for all $i \in I$, there exists a trajectory $\left(w_{1}^{i}, w_{2}^{i}, x^{i}, y^{i}\right): E \rightarrow W_{1}^{i} \times W_{2}^{i} \times X^{i} \times Y^{i} \in \mathcal{T}^{i}$ of $\Sigma^{i}$ with $w_{1}=\left(w_{1}^{1}, \ldots, w_{1}^{N}\right)$ and such that the internal inputs are constrained by the relation

$$
w_{2}^{i}(t)=\left(y^{j_{1}}(t), \ldots, y^{j_{p}}(t)\right) \text { where } \mathcal{N}(i)=\left\{j_{1}, \ldots, j_{p}\right\}
$$

for all $i \in I$ and for all $t \in E$.

By abuse of notation, the constraint on the internal inputs will be written as $w_{2}^{i}=\prod_{j \in \mathcal{N}(i)}\left\{y^{j}\right\}$.

Remark 2 Let us remark that in the proposed interconnection structure, all the internal inputs of a system are outputs of other systems. Then, the composed system $\Gamma=$ 

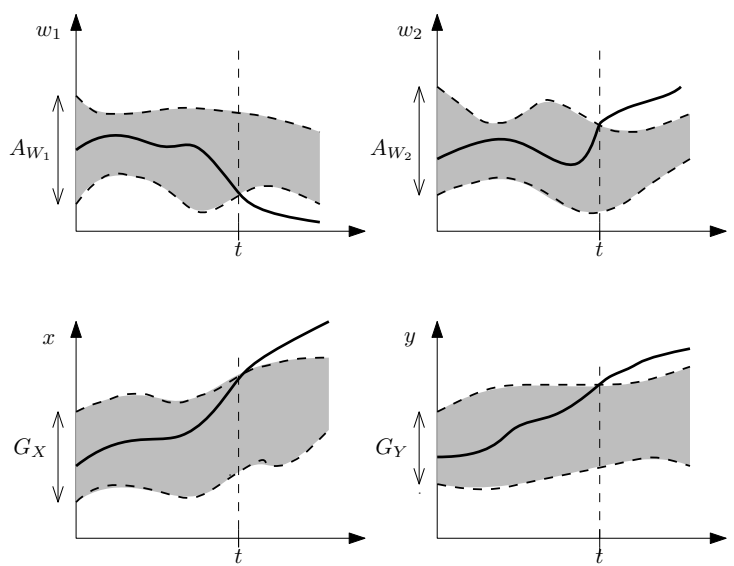

Fig. 2. An illustration of the weak satisfaction of a contract. Top: trajectories of the external $w_{1}$ and internal $w_{2}$ inputs. Bottom: trajectories of the state $x$ and output $y$.

$\left\langle\left(\Sigma^{i}\right)_{i \in I}, \mathcal{I}\right\rangle$ has trivial null internal inputs. Hence, with an abuse of notation, we will denote $\Gamma=\left(W_{1}, X, Y, \mathcal{T}\right)$ and $\left(w_{1}, x, y\right) \in \mathcal{T}$, with $\left(w_{1}, x, y\right): E \rightarrow W_{1} \times X \times Y$ as a trajectory of $\Gamma$. Similarly, all initial elements $\Sigma_{i}$, where $i \in I_{\text {init }}$ have trivial null internal inputs and we use the same notation for their trajectories.

We should emphasize that trajectories of systems need not be defined for all time, $\mathbb{R}_{0}^{+}$. This makes it possible to avoid forward-completeness issues related to systems composition as shown in the following example.

Example 5 Let us consider the system $\Sigma^{1}=\left(W_{1}, W_{2}, X\right.$, $Y, \mathcal{T})$ where $W_{1}=\{0\}, W_{2}=X=Y=\mathbb{R}$. A trajectory of $\Sigma^{1}$ is a quadruple $\left(0, w_{2}, x, y\right): E \rightarrow W_{1} \times W_{2} \times X \times Y$ in $\mathcal{T}$ where $E \in \mathbb{E}\left(\mathbb{R}_{0}^{+}\right)$, w $w_{2}$ is continuous, $x$ and $y$ are differentiable and such that $x(0)=1$ and for all $t \in E$,

$$
\left\{\begin{array}{l}
\dot{x}(t)=w_{2}(t) \\
y(t)=(x(t))^{2}
\end{array}\right.
$$

Let $I=\{1\}$ and the interconnection relation $\mathcal{I}=$ $\{(1,1)\}$. It is clear that $\left\{\Sigma^{i}\right\}_{i \in I}$ is compatible for composition with respect to $\mathcal{I}$. It can be seen that $\Sigma^{1}$, has trajectories defined on the whole time domain $\mathbb{R}_{0}^{+}$. However, if we only consider those trajectories, the set of trajectories $\mathcal{T}_{\Gamma}$ of the composed system $\Gamma=\left\langle\left(\Sigma^{i}\right)_{i \in I}, \mathcal{I}\right\rangle$ would be empty since the trajectories of $\mathcal{T}_{\Gamma}$ are of the form $(0, x, y): E \rightarrow W_{1} \times X \times Y$ where $E \subseteq[0,1)$, and for all $t \in E, x(t)=\frac{1}{1-t}$ and $y(t)=\frac{1}{(1-t)^{2}}$.

\section{Assume-guarantee contracts}

An assume-guarantee contract is a compositional tool that specifies how a system behaves under assumptions about its inputs [6]. The use of assume-guarantee contracts makes it possible to reason on a global system
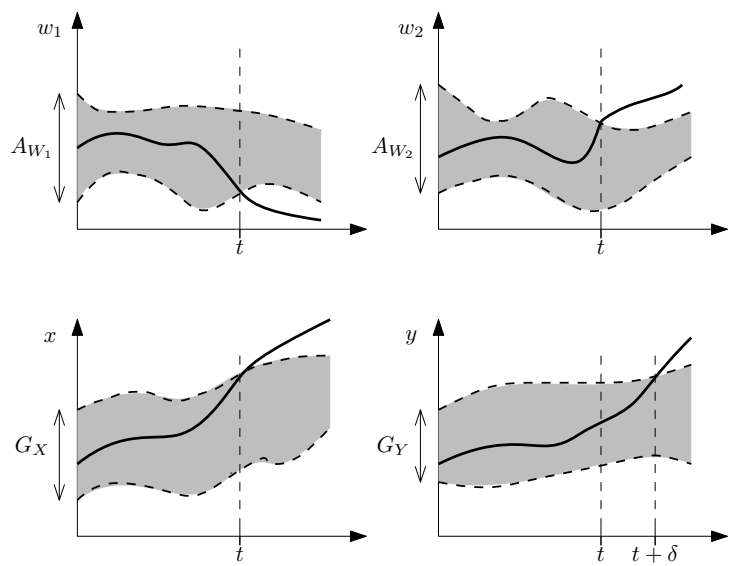

Fig. 3. An illustration of the strong satisfaction of a contract. Top: trajectories of the external $w_{1}$ and internal $w_{2}$ inputs. Bottom: trajectories of the state $x$ and output $y$.

based on properties of its components. In this section, we introduce assume-guarantee contracts to reason on properties for continuous-time systems. These contracts are equipped with a weak and a strong semantics, which will allow us to establish compositionality results.

We define contracts for continuous-time systems as follows:

Definition 5 Let $\Sigma=\left(W_{1}, W_{2}, X, Y, \mathcal{T}\right)$ be a continuoustime system, an assume-guarantee contract for $\Sigma$ is a tuple $\mathcal{C}=\left(A_{W_{1}}, A_{W_{2}}, G_{X}, G_{Y}\right)$ where

- $A_{W_{1}} \subseteq M\left(W_{1}\right)$ and $A_{W_{2}} \subseteq M\left(W_{2}\right)$ are sets of assumptions on the external and internal inputs;

- $G_{X} \subseteq M(X)$ and $G_{Y} \subseteq M(Y)$ are sets of guarantees on the states and outputs.

We say that $\Sigma$ (weakly) satisfies $\mathcal{C}$, denoted $\Sigma \models \mathcal{C}$, if for all trajectories $\left(w_{1}, w_{2}, x, y\right): E \rightarrow W_{1} \times W_{2} \times X \times Y$ in $\mathcal{T}$ :

- for all $t \in E$, if $w_{1 \mid[0, t]} \in A_{W_{1}}$ and $w_{2 \mid[0, t]} \in A_{W_{2}}$, then: - $x_{\mid[0, t]} \in G_{X}$

- $y_{\mid[0, t]} \in G_{Y}$.

We say that $\Sigma$ strongly satisfies $\mathcal{C}$, denoted $\Sigma \models{ }_{s} \mathcal{C}$, if for all trajectories $\left(w_{1}, w_{2}, x, y\right): E \rightarrow W_{1} \times W_{2} \times X \times Y$ in $\mathcal{T}$ :

- if $w_{1 \mid[0,0]} \in A_{W_{1}}$ then $y_{\mid[0,0]} \in G_{Y}$

- for all $\in E$, if $w_{1 \mid[0, t]} \in A_{W_{1}}$ and $w_{2 \mid[0, t]} \in A_{W_{2}}$, then: - $x_{\mid[0, t]} \in G_{X}$

- there exists $\delta>0$ such that for all $s \in[0, \delta]$ $y_{\mid[0, t+s] \cap E} \in G_{Y}$.

$\Sigma \models{ }_{s} \mathcal{C}$ obviously implies $\Sigma \models \mathcal{C}$. An assume-guarantee contract for a continuous-time system states that if the 
restriction of the external and internal inputs to the system up to a time $t \in \mathbb{R}_{0}^{+}$belongs to $A_{W_{1}}$ and $A_{W_{2}}$, respectively, then the restriction of the state of the system up to time $t$ belongs to $G_{X}$, and the restriction of the output of the system up to time $t$ (or up to a time $t+s$ with $s \in[0, \delta]$ and $\delta>0$, in the case of strong satisfaction) belongs to $G_{Y}$. We must highlight that the value of $\delta$ may depend on the trajectory $\left(w_{1}, w_{2}, x, y\right) \in \mathcal{T}$ and on the value of the time instant $t \in E$. An illustration of the weak and strong satisfactions is given in Figures 2 and 3. One may remark that if the set of guarantees on the outputs $G_{Y}$ is prefix closed, the notion of strong satisfaction of a contract can be defined by: $\Sigma$ strongly satisfies $\mathcal{C}$, if for all trajectories $\left(w_{1}, w_{2}, x, y\right): E \rightarrow W_{1} \times W_{2} \times X \times Y$ in $\mathcal{T}$ :

- if $w_{1 \mid[0,0]} \in A_{W_{1}}$ then $y_{\mid[0,0]} \in G_{Y}$

- for all $t \in E$, if $w_{1 \mid[0, t]} \in A_{W_{1}}$ and $w_{2 \mid[0, t]} \in A_{W_{2}}$, then: - $x_{\mid[0, t]} \in G_{X}$;

- there exists $\delta>0$ such that $y_{\mid[0, t+\delta] \cap E} \in G_{Y}$.

Remark 3 Similarly to Remark 2, a contract for the composed system $\Gamma=\left\langle\left(\Sigma^{i}\right)_{i \in I}, \mathcal{I}\right\rangle$ has trivial null assumptions on internal inputs. Hence, with an abuse of notation, a contract for the composed system $\Gamma$ will be denoted $\mathcal{C}=\left(A_{W_{1}}, G_{X}, G_{Y}\right)$.

\section{Compositional reasoning}

In this section we provide results allowing us to reason about interconnected systems based on contracts satisfied by the components.

\subsection{Acyclic interconnections}

We first provide the following result on the composition of assume-guarantee contracts, where the interconnection graph $\mathcal{G}$ between the components is a DAG.

Theorem 1 Consider a network of continuous-time components $\left\{\Sigma^{i}\right\}_{i \in I}$ compatible for composition with respect to $\mathcal{I}$. Let $\Gamma=\left\langle\left(\Sigma^{i}\right)_{i \in I}, \mathcal{I}\right\rangle$ be the composed system and assume that $\mathcal{G}=(I, \mathcal{I})$ is a DAG. To each component $\Sigma^{i}$ we associate a contract $\mathcal{C}^{i}=\left(A_{W_{1}^{i}}, A_{W_{2}^{i}}, G_{X^{i}}, G_{Y^{i}}\right)$, and let $\mathcal{C}=\left(\prod_{i \in I} A_{W_{1}^{i}}, \prod_{i \in I} G_{X^{i}}, \prod_{i \in I}^{2} G_{Y^{i}}\right)$ be a contract for $\Gamma$. If for all $i \in I, \Sigma^{i} \models \mathcal{C}^{i}$ and $\prod_{j \in \mathcal{N}(i)} G_{Y^{j}} \subseteq A_{W_{2}^{i}}$ then $\Gamma \models \mathcal{C}$.

PROOF. Let $\left(w_{1}, x, y\right): E \rightarrow W_{1} \times X \times Y$ in $\mathcal{T}$ be a trajectory of the system $\Gamma$. Then, for all $i \in I$, there exists a trajectory $\left(w_{1}^{i}, w_{2}^{i}, x^{i}, y^{i}\right): E \rightarrow W_{1}^{i} \times W_{2}^{i} \times X^{i} \times$ $Y^{i} \in \mathcal{T}^{i}$ of $\Sigma^{i}$ such that $w_{2}^{i}=\prod_{j \in \mathcal{N}(i)}\left\{y^{j}\right\}$. Let $t \in E$ such that $w_{1 \mid[0, t]} \in A_{W_{1}}$. Then we have for all $i \in I$, $w_{1 \mid[0, t]}^{i} \in A_{W_{1}^{i}}$. Since initial components $\left\{\Sigma^{i}\right\}_{i \in I_{\text {init }}}$ do not have internal inputs, and from the satisfaction of contracts for all components, we have:

$$
\forall i \in I_{\text {init }}, \quad x_{\mid[0, t]}^{i} \in G_{X^{i}} \text { and } y_{\mid[0, t]}^{i} \in G_{Y^{i}} .
$$

Let us assume the existence of $i \in I \backslash I_{\text {init }}$, such that $x_{\mid[0, t]}^{i} \notin G_{X^{i}}$ or $y_{\mid[0, t]}^{i} \notin G_{Y^{i}}$. Since $\Sigma^{i} \models \mathcal{C}^{i}$ and $w_{1 \mid[0, t]}^{i} \in$ $A_{W_{1}^{i}}$, we have that $w_{2 \mid[0, t]}^{i} \notin A_{W_{2}^{i}}$, then using the fact that $w_{2}^{i}=\prod_{j \in \mathcal{N}(i)}\left\{y^{j}\right\}$ and $\prod_{j \in \mathcal{N}(i)} G_{Y^{j}} \subseteq A_{W_{2}^{i}}$, we have the existence of $j \in \mathcal{N}(i)$ such that $y_{\mid[0, t]}^{j} \notin G_{Y^{j}}$. Hence, using the structure of a DAG, we have by iterating this procedure, the existence of $k \in I_{\text {init }}$ such that $y_{\mid[0, t]}^{k} \notin G_{Y^{k}}$ which contradicts (1). Hence, we have for all $i \in I, x_{\mid[0, t]}^{i} \in G_{X^{i}}$ and $y_{\mid[0, t]}^{i} \in G_{Y^{i}}$, which implies that $x_{\mid[0, t]} \in G_{X}$ and $y_{\mid[0, t]} \in G_{Y}$. Then, $\Gamma \models \mathcal{C}$.

Remark 4 To analyse the tightness of the composition of contracts operator, we start from a collection of local contracts $\mathcal{C}^{i}=\left(A_{W_{1}^{i}}, A_{W_{2}^{i}}, G_{X^{i}}, G_{Y^{i}}\right), i \in I$, and we proceed as follows:

- To each contract $\mathcal{C}^{i}$ we can associate a component $\Sigma^{i}=$ $\left(W_{1}^{i}, W_{2}^{i}, X^{i}, Y^{i}, \mathcal{T}^{i}\right)$, where the set $\mathcal{T}^{i}$ is the set of all possible trajectories that weakly satisfy the contract $\mathcal{C}^{i}$, i.e,

$$
\begin{gathered}
\mathcal{T}^{i}=\left\{\left(w_{1}^{i}, w_{2}^{i}, x^{i}, y^{i}\right): E \rightarrow W_{1}^{i} \times W_{2}^{i} \times X^{i} \times Y^{i} \mid\right. \\
\forall t \in E, w_{1 \mid[0, t]}^{i} \in A_{W_{1}} \text { and } w_{2 \mid[0, t]}^{i} \in A_{W_{2}} \\
\left.\Longrightarrow x_{\mid[0, t]}^{i} \in G_{X^{i}} \text { and } y_{\mid[0, t]}^{i} \in G_{Y^{i}}\right\}
\end{gathered}
$$

Consider the directed acyclic interconnection graph $\mathcal{G}=(I, \mathcal{I})$ and let $\Gamma=\left\langle\left(\Sigma^{i}\right)_{i \in I}, \mathcal{I}\right\rangle=\left(W_{1}, X, Y, \mathcal{T}_{\Gamma}\right)$ be the composed system;

- We construct the global contract $\mathcal{C}=\left(\prod_{i \in I} A_{W_{1}^{i}}\right.$, $\left.\prod_{i \in I} G_{X^{i}}, \prod_{i \in I} G_{Y^{i}}\right)$ resulting from the interconnection of the local contracts $\mathcal{C}^{i}=\left(A_{W_{1}^{i}}, A_{W_{2}^{i}}, G_{X^{i}}, G_{Y^{i}}\right)$, $i \in I$. To the contract $\mathcal{C}$, we associate the system $\Sigma=\left(W_{1}, X, Y, \mathcal{T}_{\Sigma}\right)$ where the set $\mathcal{T}_{\Sigma}$ is the set of all possible trajectories that weakly satisfy the contract $\mathcal{C}$, i.e,

$$
\begin{aligned}
& \mathcal{T}_{\Sigma}=\left\{\left(w_{1}, x, y\right): E \rightarrow W_{1} \times X \times Y \mid \forall t \in E\right. \\
& \left.w_{1 \mid[0, t]} \in A_{W_{1}} \Longrightarrow x_{\mid[0, t]} \in G_{X} \text { and } y_{\mid[0, t]} \in G_{Y}\right\}
\end{aligned}
$$

First, one can see that $\mathcal{T}_{\Gamma} \subseteq \mathcal{T}_{\Sigma}$ and, in general, one may have that $\mathcal{T}_{\Gamma} \neq \mathcal{T}_{\Sigma}$. Moreover, one can also check that the proposed contracts composition operator is tight, in the sense that the system $\Sigma$ is the smallest system defining an assume-guarantee contract and containing the trajectories of the system $\Gamma$.

\subsection{Cyclic interconnections}

In order to deal with cyclic interconnections, we need the following assumption on the set of guarantees on the 
output $G_{Y}$. This assumption will be explained later on different examples.

Assumption 1 Let $\Sigma=\left(W_{1}, W_{2}, X, Y, \mathcal{T}\right)$ be a continuous-time system, and $\mathcal{C}=\left(A_{W_{1}}, A_{W_{2}}, G_{X}, G_{Y}\right)$ an assume-guarantee contracts for $\Sigma$. For any trajectory $\left(w_{1}, w_{2}, x, y\right): E \rightarrow W_{1} \times W_{2} \times X \times Y$ of the system $\Sigma$, the following logical implication is satisfied for all $t \in E$ :

$$
\forall s \in[0, t),\left(y_{\mid[0, s]} \in G_{Y} \Rightarrow y_{\mid[0, t]} \in G_{Y}\right) .
$$

Now we give some sufficient conditions on systems and contracts in order to satisfy Assumption 1 for different examples. Let $\Sigma=\left(W_{1}, W_{2}, X, Y, \mathcal{T}\right)$ be a system, and $\mathcal{C}=\left(A_{W_{1}}, A_{W_{2}}, G_{X}, G_{Y}\right)$ an assume-guarantee contract for $\Sigma$.

- If the set $G_{Y}$ is of the same form as the set $A$ defined in Example 1 and if for any trajectory $\left(w_{1}, w_{2}, x, y\right)$ : $E \rightarrow W_{1} \times W_{2} \times X \times Y$ for the system $\Sigma, y: E \rightarrow Y$ is left continuous and the set of guarantees $G_{Y}$ is closed then Assumption 1 is satisfied.

- If the set $G_{Y}$ is of the same form as the set $A$ defined in Example 2 and similarly to the previous example, it can be shown that if for any trajectory $\left(w_{1}, w_{2}, x, y\right)$ : $E \rightarrow W_{1} \times W_{2} \times X \times Y$ for the system $\Sigma, y: E \rightarrow Y$ is $q$ times differentiable, the $q$ th derivative $y^{(q)}$ is left continuous and the sets $S_{i}, i=1, \ldots, q$, are closed then Assumption 1 is satisfied.

- If the set $G_{Y}$ is of the same form as the set $A$ defined in Example 3 and if the set valued map $F: \mathbb{R}^{n} \rightarrow 2^{\mathbb{R}^{n}}$ is outer semicontinuous (see Definition 5.4 in [31]) and for any trajectory $\left(w_{1}, w_{2}, x, y\right): E \rightarrow W_{1} \times W_{2} \times X \times$ $Y$ of the system $\Sigma, y: I \rightarrow Y$ is differentiable and its derivative is left continuous, then Assumption 1 is satisfied.

The following result relates the satisfaction of Assumption 1 for a global system to its satisfaction for the components.

Claim 1 Given a collection of components $\left\{\Sigma^{i}\right\}_{i \in I}$, such that each component $\Sigma^{i}$ satisfies Assumption 1 with respect to the contract $\mathcal{C}^{i}=\left(A_{W_{1}^{i}}, A_{W_{2}^{i}}, G_{X^{i}}, G_{Y^{i}}\right)$. Then the composed system $\Gamma=\left\langle\left(\Sigma^{i}\right)_{i \in I}, \mathcal{I}\right\rangle$ satisfies Assumption 1 with respect to the contract $\mathcal{C}=\left(\prod_{i \in I} A_{W_{1}^{i}}, \prod_{i \in I} G_{X^{i}}, \prod_{i \in I} G_{Y^{i}}\right)$.

PROOF. Let $\left(w_{1}, x, y\right): E \rightarrow W_{1} \times X \times Y$ in $\mathcal{T}$ be a trajectory of the interconnected system $\Gamma$. Let $t \in E$ and assume that for all $s \in[0, t), y_{\mid[0, s]} \in G_{Y}$. Then we have for all $s \in[0, t)$ and for all $i \in I, y_{\mid[0, s]}^{i} \in G_{Y^{i}}$. Since each component $\Sigma^{i}$ satisfies Assumption 1 with respect to the contract $\mathcal{C}^{i}=\left(A_{W_{1}^{i}}, A_{W_{2}^{i}}, G_{X^{i}}, G_{Y^{i}}\right)$, we have for all $i \in I, y_{\mid[0, t]}^{i} \in G_{Y^{i}}$, which in turn implies that $y_{\mid[0, t]} \in G_{Y}$.
Theorem 2 Consider a network of continuous-time components $\left\{\Sigma^{i}\right\}_{i \in I}$ compatible for composition with respect to $\mathcal{I}$. Let the system $\Gamma=\left\langle\left(\Sigma^{i}\right)_{i \in I}, \mathcal{I}\right\rangle$ be the composed system. To each component $\Sigma^{i}$, we associate a contract $\mathcal{C}^{i}=\left(A_{W_{1}^{i}}, A_{W_{2}^{i}}, G_{X^{i}}, G_{Y^{i}}\right)$ and let $\mathcal{C}=\left(\prod_{i \in I} A_{W_{1}^{i}}, \prod_{i \in I} G_{X^{i}}, \prod_{i \in I}^{1} G_{Y^{i}}\right)$ be a contract for $\Gamma$. Let us assume the following:

(i) for all $i \in I, \Sigma^{i} \models \mathcal{C}^{i}$;

(ii) for all $i \in I, \prod_{j \in \mathcal{N}(i)} G_{Y^{j}} \subseteq A_{W_{2}^{i}}$;

(iii) for all $i \in I, \Sigma^{i}$ satisfies Assumption 1;

(iv) for any cycle $\zeta_{q}$ in the interconnection graph $\mathcal{G}=$ $(I, \mathcal{I})$, there exists an element $k \in \zeta_{q}$ such that $\Sigma^{k} \models_{s}$ $\mathcal{C}^{k}$

(v) for all $i \in I, A_{W_{1}^{i}}$ is a prefix-closed set.

then $\Gamma \models \mathcal{C}$.

PROOF. Let $\left(w_{1}, x, y\right): E \rightarrow W_{1} \times X \times Y$ in $\mathcal{T}$ be a trajectory of the system $\Gamma$. Then, for all $i \in I$, there exists a trajectory $\left(w_{1}^{i}, w_{2}^{i}, x^{i}, y^{i}\right): E \rightarrow W_{1}^{i} \times W_{2}^{i} \times$ $X^{i} \times Y^{i} \in \mathcal{T}^{i}$ of $\Sigma^{i}$ such that $w_{2}^{i}=\prod_{j \in \mathcal{N}(i)}\left\{y^{j}\right\}$. Let $t \in E$ such that $w_{1 \mid[0, t]} \in A_{W_{1}}$. Then we have for all $i \in I, w_{1 \mid[0, t]}^{i} \in A_{W_{1}^{i}}$. All initial components $\left\{\Sigma^{i}\right\}_{i \in I_{\text {init }}}$ do not have internal inputs, then from the satisfaction of contracts for all components and since $A_{W_{1}^{i}}$ is prefixclosed for all $i \in I$, we have:

$$
\forall i \in I_{\text {init }}, \quad \forall s \in[0, t], \quad x_{\mid[0, s]}^{i} \in G_{X^{i}} \text { and } y_{\mid[0, s]}^{i} \in G_{Y^{i}} \text {. }
$$

First, let us prove that

$$
\forall i \in I, \quad y_{\mid[0,0]}^{i} \in G_{Y^{i}}
$$

We have the existence of an element $k$ in any cycle $\zeta_{q}$ such that $\Sigma^{k}={ }_{s} \mathcal{C}^{k}$, which implies from prefix closedeness of $A_{W_{1}^{k}}$ that $y_{\mid[0,0]}^{k} \in G_{Y^{k}}$. To prove that this initial condition is satisfied by all the components $\Sigma^{i}, i \in I$, we proceed as follows: for any component $\Sigma^{k}$ that strongly satisfies its contract, we drop the incoming edge into the vertex $k$ in the cycle $\zeta_{q}$. Then, in view of Remark 1 , a new DAG, $\mathcal{G}^{\mathrm{DAG}}$ is obtained. Then from (2) we have:

$$
\forall i \in I_{\text {init }}^{\mathrm{DAG}} \subseteq I_{\text {init }} \cup A, \quad y_{\mid[0,0]}^{i} \in G_{Y^{i}}
$$

where $A$ is the set of vertices to which we dropped an edge (vertices corresponding to components that strongly satisfy their contracts). Now let an element $i \in I \backslash I_{\text {init }}^{\mathrm{DA}}$ and let us assume that $y_{\mid[0,0]}^{i} \notin G_{Y^{i}}$. From prefix-closedness of $A_{W_{1}^{i}}$ it follows that $w_{1 \mid[0,0]}^{i} \in A_{W_{1}^{i}}$, moreover $\Sigma^{i} \models \mathcal{C}^{i}$, then we have that $w_{2 \mid[0,0]}^{i} \notin A_{W_{2}^{i}}$, and using the fact that $w_{2}^{i}=\prod_{j \in \mathcal{N}(i)}\left\{y^{j}\right\}$ and $\prod_{j \in \mathcal{N}(i)} G_{Y^{j}} \subseteq A_{W_{2}^{i}}$, we have the existence of $j \in \mathcal{N}(i)$ such that $y_{\mid[0,0]}^{j} \notin G_{Y^{j}}$. 
Hence, using the structure of a DAG, we have by iterating this procedure, the existence of $h \in I_{\text {init }}^{\mathrm{DAG}}$ such that $y_{\mid[0,0]}^{h} \notin G_{Y^{h}}$ which contradicts (4). Hence, we have for all $i \in I, y_{\mid[0,0]}^{i} \in G_{Y^{i}}$, which implies that $y_{\mid[0,0]} \in G_{Y}$. To prove that $\Gamma \models \mathcal{C}$, we proceed by contradiction. Let us define

$$
\begin{aligned}
T & =\sup \left\{s \in[0, t] \mid \forall s^{\prime} \in[0, s], y_{\mid\left[0, s^{\prime}\right]} \in G_{Y}\right\} \\
& =\sup \left\{s \in[0, t] \mid \forall i \in I, \forall s^{\prime} \in[0, s], y_{\mid\left[0, s^{\prime}\right]}^{i} \in G_{Y^{i}}\right\} .
\end{aligned}
$$

From (3) we have $y_{\mid[0,0]} \in G_{Y}$, it then follows that $T \in$ $[0, t]$. Let us remark that by $(5)$, we have that $y_{\mid[0, s]} \in G_{Y}$ for all $s \in[0, T)$.

We have $y_{\mid[0, s]} \in G_{Y}$ for all $s \in[0, T)$. Then, from (iii) and using Claim 1, we have that $y_{\mid[0, T]} \in G_{Y}$. We have the existence of an element $k$ in any cycle $\zeta_{q}$ such that $\Sigma^{k} \models{ }_{s} \mathcal{C}^{k}$. We have from prefix-closedeness of the set $A_{W_{1}^{k}}$ that $w_{1 \mid[0, T]}^{k} \in A_{W_{1}^{k}}$. Then, since

$$
w_{2 \mid[0, T]}^{k}=\prod_{j \in \mathcal{N}(k)}\left\{y_{\mid[0, T]}^{j}\right\} \in \prod_{j \in \mathcal{N}(k)} G_{Y^{j}} \subseteq A_{W_{2}^{k}}
$$

we have from (iv) the existence of $\delta_{k}>0$ such that

$$
\forall s_{k} \in\left[0, \delta_{k}\right], y_{\mid\left[0, T+s_{k}\right] \cap E}^{k} \in G_{Y^{k}}
$$

Let $\delta=\min _{k \in A} \delta_{k}$, where $A$ is the set of vertices corresponding to components that strongly satisfy their contracts, we have that

$$
\forall s \in[0, \delta], y_{\mid[0, T+s] \cap E}^{k} \in G_{Y^{k}}
$$

by using the same procedure as above (dropping the incoming edges into the vertex $k$ in the cycle $\zeta_{q}$ ), we have from (2):

$\forall i \in I_{\text {init }}^{\mathrm{DAG}} \subseteq I_{\text {init }} \cup A, \quad \forall s \in[0, \delta], \quad y_{\mid[0, T+s] \cap[0, t]}^{i} \in G_{Y^{i}}$

Now let an element $i \in I \backslash I_{\mathrm{init}}^{\mathrm{DAG}}$ and let us assume the existence of $s^{\prime} \in[0, \delta]$ such that $y_{\mid\left[0, T+s^{\prime}\right] \cap[0, t]}^{i} \notin G_{Y^{i}}$. From prefix-closedeness of $A_{W_{1}^{i}}$ we have that $w_{1 \mid\left[0, T+s^{\prime}\right] \cap[0, t]}^{i} \in$ $A_{W_{1}^{i}}$. Then, since $\Sigma^{i} \models \mathcal{C}^{i}$ we have that $w_{2 \mid\left[0, T+s^{\prime}\right] \cap[0, t]}^{i} \notin$ $A_{W_{2}^{i}}$, then using the fact that $w_{2}^{i}=\prod_{j \in \mathcal{N}(i)}\left\{y^{j}\right\}$ and $\prod_{j \in \mathcal{N}(i)} G_{Y^{j}} \subseteq A_{W_{2}^{i}}$, we have the existence of $j \in \mathcal{N}(i)$ such that $y_{\mid\left[0, T+s^{\prime}\right] \cap[0, t]}^{j} \notin G_{Y^{j}}$. Hence, using the structure of a DAG, we have by iterating this procedure, the existence of $h \in I_{\text {init }}^{\mathrm{DAG}}$ such that $y_{\mid\left[0, T+s^{\prime}\right] \cap[0, t]}^{h} \notin G_{Y^{h}}$ which contradicts (6). Hence, we have

$$
\forall i \in I, \forall s \in[0, \delta], y_{\mid[0, T+s] \cap[0, t]}^{i} \in G_{Y^{i}}
$$

which by definition of $T$ implies that $T=t$. Hence, we have for all $i \in I$ and for all $s \in[0, t], y_{\mid[0, s]}^{i} \in G_{Y^{i}}$, which implies that $y_{\mid[0, s]} \in G_{Y}$ for all $s \in[0, t]$.

Let $i \in I$, we have $w_{1 \mid[0, t]}^{i} \in A_{W_{1}^{i}}$ and

$$
w_{2 \mid[0, t]}^{i}=\prod_{j \in \mathcal{N}(i)}\left\{y_{\mid[0, t]}^{i}\right\} \in \prod_{j \in \mathcal{N}(i)} G_{Y^{j}} \subseteq A_{W_{2}^{i}}
$$

then we have from (i) that for all $i \in I, x_{\mid[0, t]}^{i} \in G_{X^{i}}$, which implies that $x_{\mid[0, t]} \in G_{X}$. Hence, $\Gamma \models \mathcal{C}$.

The proof of Theorem 2 can be interpreted as follows: from the satisfaction of contracts, the time interval on which the property holds for the output may be rightopen $[0, T)$. Then from Assumption 1, we can ensure that the property holds on its closure $[0, T]$. Hence, one can use the strong satisfaction again to move further and to enlarge the time interval on which the property holds $[0, T+\delta]$. Thus, it has to be the whole time domain on which the trajectory is defined.

Remark 5 It was shown in Theorem 2 that prefixclosedeness of the set of assumptions $A_{W_{1}}$ is critical for the compositionality result for general interconnections containing cycles. Given a non prefix-closed set of assumptions $A_{W_{1}}$, the set pref $\left(A_{W_{1}}\right)$ is prefixclosed. Hence, the result of Theorem 2 remain correct if we assign to each component $\Sigma^{i}$ the contract $\mathcal{C}^{i}=\left(\operatorname{pref}\left(A_{W_{1}^{i}}\right), A_{W_{2}^{i}}, G_{X^{i}}, G_{Y^{i}}\right)$. This approach allows to overcome the prefix-closedeness of the set of assumptions on the external inputs $A_{W_{1}}$, at the cost of an additional conservatism.

Remark 6 Proposition 1 in [29] can be recovered by the result of Theorem 2, where our prefix-closed sets and general sets corresponds to invariants and LTL specifications, respectively, in that work.

Let us point out that weak satisfaction is generally insufficient to reason about general compositions containing cycles, as shown by the following counter-example:

Example 6 Let us consider the system $\Sigma^{1}=\left(W_{1}, W_{2}, X\right.$, $Y, \mathcal{T})$ where, $W_{1}=W_{2}=X=Y=\mathbb{R}_{0}^{+}$. A trajectory of $\Sigma^{1}$ is a quadruple $\left(w_{1}, w_{2}, x, y\right): E \rightarrow W_{1} \times W_{2} \times X \times Y$ in $\mathcal{T}$ where $E \in \mathbb{E}\left(\mathbb{R}_{0}^{+}\right), w_{1}$ and $w_{2}$ are continuous, $x$ and $y$ are differentiable and such that $x(0)=0$, and for all $t \in \mathbb{R}_{0}^{+}$,

$$
\left\{\begin{array}{l}
\dot{x}(t)=\sqrt{w_{2}(t)}+w_{1}(t) \\
y(t)=x(t)
\end{array}\right.
$$

Let $I=\{1\}$ and consider the interconnection relation $\mathcal{I}=\{(1,1)\}$. It is clear that $\left\{\Sigma^{i}\right\}_{i \in I}$ is compatible for 
composition with respect to $\mathcal{I}$. Let us consider the assumeguarantee contract $\mathcal{C}=\left(A_{W_{1}}, A_{W_{2}}, G_{X}, G_{Y}\right)$ for $\Sigma^{1}$, given by:

$$
\begin{aligned}
A_{W_{1}} & =\left\{w_{1}: E \rightarrow W_{1} \in M\left(W_{1}\right) \mid \forall t \in E, w_{1}(t)=0\right\} \\
A_{W_{2}} & =\left\{w_{2}: E \rightarrow W_{2} \in M\left(W_{2}\right) \mid \forall t \in E, w_{2}(t)=0\right\} \\
G_{X} & =\{x: E \rightarrow X \in M(X) \mid \forall t \in E, x(t)=0\} \\
G_{Y} & =\{y: E \rightarrow Y \in M(Y) \mid \forall t \in E, y(t)=0\}
\end{aligned}
$$

Let $\mathcal{C}_{\Gamma}$ be the contract for the composed system $\Gamma=$ $\left\langle\left(\Sigma^{i}\right)_{i \in I}, \mathcal{I}\right\rangle$ defined as in Theorem 2. We can easily check that $\Sigma^{1}=\mathcal{C}$ and that Assumption 1 holds. However, the conclusion of the previous theorem does not hold. Indeed, the map $\left(w_{1}, x, y\right): \mathbb{R}_{0}^{+} \rightarrow W_{1} \times X \times Y$ defined by $w_{1}(t)=0$ and $x(t)=y(t)=t^{2} / 4$ for all $t \in \mathbb{R}_{0}^{+}$is a trajectory of $\Gamma$ and the contract $\mathcal{C}_{\Gamma}$ of the system $\Gamma$ is not satisfied.

It is clear from the previous example that strong satisfaction is needed to reason about general interconnections containing cycles. We show two modifications of the previous example, based on sampling or time-delays, which lead to strong satisfaction of the contract.

Example 7 Consider the system $\Sigma^{1}=\left(W_{1}, W_{2}, X, Y, \mathcal{T}\right)$ where $W_{1}=W_{2}=X=Y=\mathbb{R}_{0}^{+}$. A trajectory of $\Sigma^{1}$ is a quadruple $\left(w_{1}, w_{2}, x, y\right): E \rightarrow W_{1} \times W_{2} \times X \times Y$ in $\mathcal{T}$ where $E \in \mathbb{E}\left(\mathbb{R}_{0}^{+}\right), w_{1}$ and $w_{2}$ are continuous, $x$ and $y$ are differentiable and such that $x(0)=0$, and for all $t \in \mathbb{R}_{0}^{+}$,

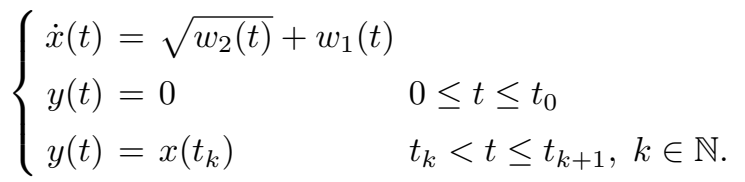

where $\left(t_{k}\right)_{k \in \mathbb{N}}$ a strictly increasing sequence of sampling instants with $t_{0} \geq 0$ and $t_{k} \rightarrow+\infty$ when $k \rightarrow+\infty$. We consider the same assume-guarantee contract as in the previous example. It can be seen that $y$ is left-continuous and Assumption 1 is satisfied. We can easily check that $\Sigma^{1}={ }_{s} \mathcal{C}$, where the value of $\delta$ as in Definition 5 is given by $\delta=t_{k+1}-t$ if $t_{k} \leq t<t_{k+1}$. Let $I=\{1\}$ and consider the interconnection relation $\mathcal{I}=\{(1,1)\}$. Let $\mathcal{C}_{\Gamma}$ be the contract for the composed system $\Gamma=\left\langle\left(\Sigma^{i}\right)_{i \in I}, \mathcal{I}\right\rangle$ defined as in Theorem 2. Now we can check that the conclusion of the previous theorem holds since the only trajectory $\left(w_{1}, x, y\right): \mathbb{R}_{0}^{+} \rightarrow W_{1} \times X \times Y$ of the composed system $\Gamma$ is given by $w_{1}(t)=x(t)=y(t)=0$, for all $t \in \mathbb{R}_{0}^{+}$.

Example 8 Consider the system $\Sigma^{1}=\left(W_{1}, W_{2}, X, Y, \mathcal{T}\right)$ where $W_{1}=W_{2}=X=Y=\mathbb{R}_{0}^{+}$. A trajectory of $\Sigma^{1}$ is a quadruple $\left(w_{1}, w_{2}, x, y\right): E \rightarrow W_{1} \times W_{2} \times X \times Y$ in $\mathcal{T}$ where $E \in \mathbb{E}\left(\mathbb{R}_{0}^{+}\right), w_{1}$ and $w_{2}$ are continuous, $x$ and $y$ are differentiable and such that $x(0)=0$, and for all $t \in \mathbb{R}_{0}^{+}$,

$$
\left\{\begin{array}{llrl}
\dot{x}(t) & =\sqrt{w_{2}(t)}+w_{1}(t) & \\
y(t) & =0 & & 0 \leq t \leq T \\
y(t) & =x(t-T) & & T<t
\end{array}\right.
$$

where $T>0$ is a time delay. We consider the same assume-guarantee contract as in Example 6. It can be seen that $y$ is left-continuous and Assumption 1 is satisfied. We can easily check that $\Sigma \models_{s} \mathcal{C}$, where the value of $\delta$ as in Definition 5 is given by $\delta=T$. Let $I=\{1\}$ and consider the interconnection relation $\mathcal{I}=\{(1,1)\}$. Let the contract $\mathcal{C}_{\Gamma}$ for the composed system $\Gamma=\left\langle\left(\Sigma^{i}\right)_{i \in I}, \mathcal{I}\right\rangle$ defined as in Theorem 2. Then, we can check that the conclusion of the previous theorem holds since the only trajectory $\left(w_{1}, x, y\right): \mathbb{R}_{0}^{+} \rightarrow W_{1} \times X \times Y$ of the composed system $\Gamma$ is given by $w_{1}(t)=x(t)=y(t)=0$, for all $t \in \mathbb{R}_{0}^{+}$.

It can be seen from the Examples 7 and 8 that our framework is suitable to reason about systems that include some sampled or delayed behaviors. Moreover, these examples suggest that by sampling or delaying the output of a component, strong satisfaction of a contract can be obtained. These examples also show how one can go from weak to strong satisfaction by slightly modifying the system. In the next section, we show that this is also possible by slightly modifying the contract.

It is noteworthy to mention that Theorem 2 does not hold in general without Assumption 1, as shown by the following counter-example.

Example 9 Consider the system $\Sigma^{1}=\left(W_{1}, W_{2}, X, Y, \mathcal{T}\right)$ where $W_{1}=W_{2}=X=Y=\mathbb{R}$. A trajectory of $\Sigma^{1}$ is a quadruple $\left(w_{1}, w_{2}, x, y\right): E \rightarrow W_{1} \times W_{2} \times X \times Y$ in $\mathcal{T}$ where $E \in \mathbb{E}\left(\mathbb{R}_{0}^{+}\right), w_{1}, w_{2}, x, y$ are continuous, $w_{2}(0)=0$ and for all $t \in \mathbb{R}_{0}^{+}$,

$$
\left\{\begin{array}{l}
x(t)=w_{2}(t)+w_{1}(t) \\
y(t)=x(t)
\end{array}\right.
$$

Let $I=\{1\}$ and consider the interconnection relation $\mathcal{I}=\{(1,1)\}$. It is clear that $\left\{\Sigma^{i}\right\}_{i \in I}$ is compatible for composition with respect to $\mathcal{I}$. Let us consider the assumeguarantee contract $\mathcal{C}=\left(A_{W_{1}}, A_{W_{2}}, G_{X}, G_{Y}\right)$ for $\Sigma^{1}$, given by:

$$
\begin{aligned}
A_{W_{1}} & =\left\{w_{1}: E \rightarrow W_{1} \in M\left(W_{1}\right) \mid \forall t \in E, w_{1}(t)=0\right\} \\
A_{W_{2}} & =\left\{w_{2}: E \rightarrow W_{2} \in M\left(W_{2}\right) \mid \forall t \in E, w_{2}(t) \in(-1,1)\right\} \\
G_{X} & =\{x: E \rightarrow X \in M(X) \mid \forall t \in E, x(t) \in(-1,1)\} \\
G_{Y} & =\{y: E \rightarrow Y \in M(Y) \mid \forall t \in E, y(t) \in(-1,1)\}
\end{aligned}
$$

Let $\mathcal{C}_{\Gamma}$ be the contract for the composed system $\Gamma=$ $\left\langle\left(\Sigma^{i}\right)_{i \in I}, \mathcal{I}\right\rangle$ defined as in Theorem 2. We can easily check 
from the continuity of $y$ that $\Sigma^{1} \models{ }_{s} \mathcal{C}$ and that all the assumptions of Theorem 2 hold except for Assumption 1. Moreover, we can check that the conclusion of Theorem 2 does not hold. Indeed, for $w_{1}: \mathbb{R}_{0}^{+} \rightarrow W_{1} \in A_{W_{1}}$ any map $\left(w_{1}, x, y\right): \mathbb{R}_{0}^{+} \rightarrow W_{1} \times X \times Y$ satisfying $x(0)=y(0)=0$ and $x(t)=y(t)$ for all $t \in \mathbb{R}_{0}^{+}$is a trajectory of $\Gamma$ and the contract $\mathcal{C}_{\Gamma}$ of the system $\Gamma$ is not satisfied.

Remark 7 Theorems 1 and 2 apply to a very general class of systems. When considering more specific classes, one can sometimes reason on general interconnections without strong contract satisfaction. Such a case will be shown in Section 6, where we consider systems modeled by Lipschitz differential inclusions and invariance assumeguarantee contracts.

\subsubsection{From weak to strong contract satisfaction}

In this section, we show that under some additional assumptions, it is possible to reason about general compositions using the weak semantics of assume guarantee contracts.

In order to measure the distance between two continuoustime trajectories, which might not have the same time domain. We use the notion of $\varepsilon$-closeness of trajectories [17], which is related to the Hausdorff distance between the graphs of the trajectories.

Definition 6 ( $\varepsilon$-closeness of trajectories) Let $Z \subseteq \mathbb{R}^{n}$. Given $\varepsilon>0$ and two continuous-time trajectories $z_{1}$ : $E_{1} \rightarrow Z$ and $z_{2}: E_{2} \rightarrow Z$ in $M(Z) . z_{2}$ is said to be $\varepsilon$-close to $z_{1}$, if for all $t_{1} \in E_{1}$, there exists $t_{2} \in E_{2}$ such that $\left|t_{1}-t_{2}\right| \leq \varepsilon$ and $\left\|z_{1}\left(t_{1}\right)-z_{2}\left(t_{2}\right)\right\| \leq \varepsilon$. We define the $\varepsilon$-expansion of $z_{1}$ by: $\mathcal{B}_{\varepsilon}\left(z_{1}\right)=\left\{z^{\prime}: \bar{E}^{\prime} \rightarrow Z \mid\right.$ $z^{\prime}$ is $\varepsilon$-close to $\left.z\right\}$.

This notion is generalized toward sets of continuoustime maps in the usual way: For $A \subseteq M(Z), \mathcal{B}_{\varepsilon}(A)=$ $\bigcup_{z \in A} \mathcal{B}_{\varepsilon}(z)$.

Proposition 2 Let $\Sigma=\left(W_{1}, W_{2}, X, Y, \mathcal{T}\right)$ be a continuous-time system and let $\mathcal{C}=\left(A_{W_{1}}, A_{W_{2}}, G_{X}, G_{Y}\right)$ be an assume-guarantee contract for $\Sigma$. Let us assume that for all trajectories $\left(w_{1}, w_{2}, x, y\right): E \rightarrow$ $W_{1} \times W_{2} \times X \times Y \in \mathcal{T}, y: E \rightarrow Y$ is continuous and $y_{\mid[0,0]} \in G_{Y}$ if $w_{1 \mid[0,0]} \in A_{W_{1}}$. If $\Sigma \models \mathcal{C}$, then for all $\varepsilon>0$, $\Sigma \models{ }_{s} \mathcal{C}_{\varepsilon}$ where $\mathcal{C}_{\varepsilon}=\left(A_{W_{1}}, A_{W_{2}}, G_{X}, \mathcal{B}_{\varepsilon}\left(G_{Y}\right) \cap M(Y)\right)$.

PROOF. Let $\left(w_{1}, w_{2}, x, y\right): E \rightarrow W_{1} \times W_{2} \times X \times$ $Y \in \mathcal{T}$, then $y_{\mid[0,0]} \in G_{Y} \subseteq \mathcal{B}_{\varepsilon}\left(G_{Y}\right) \cap M(Y)$. Let $t \in$ $E$, such that $w_{1 \mid[0, t]} \in A_{W_{1}}$ and $w_{2 \mid[0, t]} \in A_{W_{2}}$. Then, satisfaction of $\mathcal{C}$ gives that $x_{\mid[0, t]} \in G_{X}$ and $y_{\mid[0, t]} \in$ $G_{Y}$. By continuity of $y$, there exists $\delta>0$ such that for all $s \in[0, \delta], y_{\mid[0, t+s] \cap E} \in \mathcal{B}_{\varepsilon}\left(G_{Y}\right)$. Also by definition,
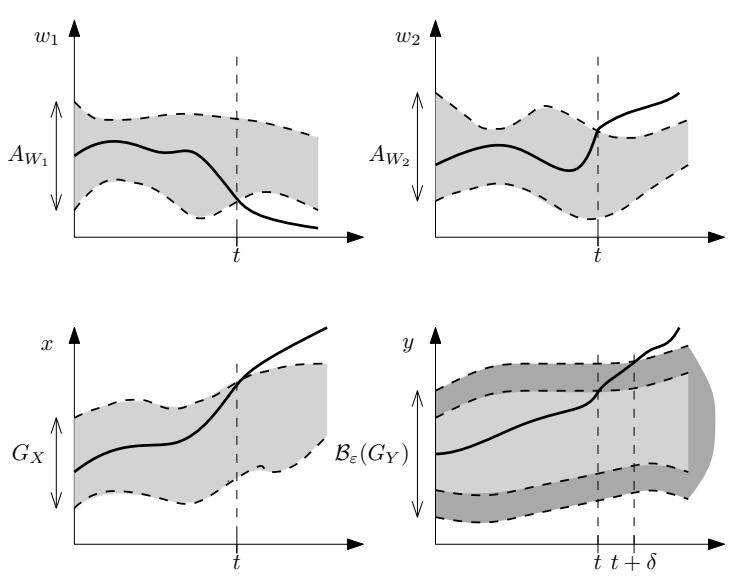

Fig. 4. An illustration of the result of Proposition 2. Top: trajectories of the external $w_{1}$ and internal $w_{2}$ inputs. Bottom: trajectories of the state $x$ and output $y$.

$y_{\mid[0, t+s] \cap E} \in M(Y)$ for all $s \in[0, \delta]$. Hence, $y_{\mid[0, t+s] \cap E} \in$ $\mathcal{B}_{\varepsilon}\left(G_{Y}\right) \cap M(Y)$, for all $s \in[0, \delta]$.

A pictural representation for the result of proposition 2 is shown in Figure 4. The following example shows an application of the previous corollary:

Example 10 Consider the system $\Sigma^{1}=\left(W_{1}, W_{2}, X, Y, \mathcal{T}\right)$ where $W_{1}=W_{2}=X=Y=\mathbb{R}_{0}^{+}$. A trajectory of $\Sigma^{1}$ is a triple $\left(w_{1}, w_{2}, x, y\right): E \rightarrow W_{1} \times W_{2} \times X \times Y$ in $\mathcal{T}$ where $E=\mathbb{R}_{0}^{+}, w_{1}$ and $w_{2}$ are continuous, $x$ and $y$ are differentiable and such that $x(0)=0$, and for all $t \in \mathbb{R}_{0}^{+}$,

$$
\left\{\begin{array}{l}
\dot{x}(t)=\sqrt{w_{2}(t)}-x(t)+w_{1}(t) \\
y(t)=x(t) .
\end{array}\right.
$$

Let $I=\{1\}$ and consider the interconnection relation $\mathcal{I}=\{(1,1)\}$. It is clear that $\left\{\Sigma^{i}\right\}_{i \in I}$ is compatible for composition with respect to $\mathcal{I}$. Let $a>1$ and let us consider the assume-guarantee contract $\mathcal{C}=\left(A_{W_{1}}, A_{W_{2}}, G_{X}, G_{Y}\right)$ for $\Sigma^{1}$, given by:

$$
\begin{aligned}
A_{W_{1}} & =\left\{w_{1}: E \rightarrow W_{1} \in M\left(W_{1}\right) \mid \forall t \in E, w_{1}(t)=0\right\} \\
A_{W_{2}} & =\left\{w_{2}: E \rightarrow W_{2} \in M\left(W_{2}\right) \mid \forall t \in E, w_{2}(t) \in\left[0, a^{2}\right]\right\} \\
G_{X} & =\{x: E \rightarrow X \in M(X) \mid \forall t \in E, x(t) \in[0, a]\} \\
G_{Y} & =\{y: E \rightarrow Y \in M(Y) \mid \forall t \in E, y(t) \in[0, a]\}
\end{aligned}
$$

We can easily check that $\Sigma^{1} \models \mathcal{C}$ and for all trajectories $\left(w_{1}, w_{2}, x, y\right) \in \mathcal{T}, y: E \rightarrow Y$ is continuous and $y_{\mid[0,0]} \in$ $G_{Y}$. Then, from Proposition 2, we have that $\Sigma \models_{s} \mathcal{C}_{\varepsilon}$ for any $\varepsilon>0$, where $\mathcal{C}_{\varepsilon}=\left(A_{W_{1}}, A_{W_{2}}, G_{X}, \mathcal{B}_{\varepsilon}\left(G_{Y}\right) \cap M(Y)\right)$. Now let $\varepsilon>0$, such that

$$
\begin{aligned}
\mathcal{B}_{\varepsilon}\left(G_{Y}\right) \cap M(Y) & =\{y: E \rightarrow Y \in M(Y) \mid \forall t \in E, y(t) \in[0, a+\varepsilon]\} \\
& \subseteq\left\{y: E \rightarrow Y \in M(Y) \mid \forall t \in E, y(t) \in\left[0, a^{2}\right]\right\} \\
& =A_{W_{2}}
\end{aligned}
$$


Then, since the system $\Sigma^{1}$ satisfies Assumption 1 (the output trajectory $y: E \rightarrow Y$ is continuous and the set $[0, a]$ is closed), we have from Theorem 2 that the composed system $\Gamma=\left\langle\left(\Sigma^{i}\right)_{i \in I}, \mathcal{I}\right\rangle$ satisfies the composed contract $\mathcal{C}_{\Gamma}=$ $\left(A_{W_{1}}, G_{X}, \mathcal{B}_{\varepsilon}\left(G_{Y}\right) \cap M(Y)\right)$. Let us remark that there exist trajectories of the composed system $\Gamma$ given by: $\left(w_{1}, x, y\right)$ : $\mathbb{R}_{0}^{+} \rightarrow W_{1} \times X \times Y$, where $w_{1}(t)=0$ and $x(t)=y(t)=$ $\left(1-e^{-t / 2}\right)^{2}$, for all $t \in \mathbb{R}_{0}^{+}$.

We have shown how one can go from weak to strong satisfaction of a contract, by relaxing the guarantees on the output. In the next result, we show that it is also possible to do so by relaxing the assumptions.

Proposition 3 Let $\Sigma=\left(W_{1}, W_{2}, X, Y, \mathcal{T}\right)$ be a continuous-time system and let $\mathcal{C}=\left(A_{W_{1}}, A_{W_{2}}, G_{X}, G_{Y}\right)$ be an assume-guarantee contract for $\Sigma$. Let us assume that for all trajectories $\left(w_{1}, w_{2}, x, y\right): E \rightarrow$ $W_{1} \times W_{2} \times X \times Y \in \mathcal{T}, w_{1}: E \rightarrow W_{1}$ and $w_{2}: E \rightarrow W_{2}$ are continuous and $y_{\mid[0,0]} \in G_{Y}$ if $w_{1 \mid[0,0]} \in A_{W_{1}}$. For an $\varepsilon>0$, if $\Sigma \models \mathcal{C}^{\varepsilon}$, with $\mathcal{C}^{\varepsilon}=\left(\mathcal{B}_{\varepsilon}\left(A_{W_{1}}\right) \cap M\left(W_{1}\right), \mathcal{B}_{\varepsilon}\left(A_{W_{2}}\right) \cap M\left(W_{2}\right), G_{X}, G_{Y}\right)$. Then, $\Sigma \mid={ }_{s} \mathcal{C}$.

PROOF. Let $\left(w_{1}, w_{2}, x, y\right): E \rightarrow W_{1} \times W_{2} \times X \times Y \in$ $\mathcal{T}$, then $y_{\mid[0,0]} \in G_{Y}$. Let $t \in E$, such that $w_{1 \mid[0, t]} \in A_{W_{1}}$ and $w_{2 \mid[0, t]} \in A_{W_{2}}$. By continuity of $w_{1}$ and $w_{2}$, there exists $\delta>0$ such that for all $s \in[0, \delta], w_{1 \mid[0, t+s] \cap E} \in$ $\mathcal{B}_{\varepsilon}\left(A_{W_{1}}\right)$ and $w_{2 \mid[0, t+s] \cap E} \in \mathcal{B}_{\varepsilon}\left(A_{W_{2}}\right)$. Also by definition, $w_{1 \mid[0, t+s] \cap E} \in M\left(W_{1}\right)$ and $w_{2 \mid[0, t+s] \cap E} \in M\left(W_{2}\right)$ for all $s \in[0, \delta]$. Then, satisfaction of $\mathcal{C}^{\varepsilon}$ gives that $x_{\mid[0, t+s]} \in$ $G_{X}$ and $y_{\mid[0, t+s]} \in G_{Y}$ for all $s \in[0, \delta]$.

We recall that the approach proposed in Proposition 3 to ensure strong satisfaction of contract has been used in [32] to construct symbolic controllers for sampleddata systems. Interestingly, this technique is useful in practice, since it allows to ensure strong satisfaction of contracts without reasoning in terms of $\delta$ which may depend on time and trajectory.

\section{Compositional invariants for differential in- clusions}

In this section, we focus on continuous-time systems $\Sigma=\left(W_{1}, W_{2}, X, Y, \mathcal{T}\right)$ defined by differential inclusions, and invariance assume-guarantee contracts, where assumptions and guarantees are defined as in Example 1. We use the classical characterization of invariant sets for differential inclusions developed using the concept of contingent cone (see [4] and the references therein) to derive necessary and sufficient conditions for weak satisfaction of assume-guarantee contracts. We also show that under some technical assumptions (Lipschitzness of the vector field and the output map), weak satisfaction makes it possible to reason on general interconnections containing cycles.

A trajectory of $\Sigma$ is a triple $\left(w_{1}, w_{2}, x, y\right): E \rightarrow W_{1} \times$ $W_{2} \times X \times Y$ in $\mathcal{T}$ where $E \in \mathbb{E}\left(\mathbb{R}_{0}^{+}\right), w_{1}$ and $w_{2}$ are locally measurable, $x$ and $y$ are absolutely continuous and continuous, respectively, and satisfy for almost all $t \in E$ :

$\left\{\begin{array}{l}\dot{x}(t) \in F\left(x(t), w_{1}(t), w_{2}(t)\right), x(0) \in X_{0} \\ y(t)=h(x(t))\end{array}\right.$

where $F: \mathbb{R}^{n} \times \mathbb{R}^{m_{1}} \times \mathbb{R}^{m_{2}} \rightarrow 2^{\mathbb{R}^{n}}$ is a set-valued map, $h: \mathbb{R}^{n} \rightarrow \mathbb{R}^{p}$ is continuous and $X_{0}$ is the set of initial conditions. Let us introduce the following assumption on the system $\Sigma$ :

Assumption 2 The set-valued $\operatorname{map}^{1} F: \mathbb{R}^{n} \times \mathbb{R}^{m_{1}} \times$ $\mathbb{R}^{m_{2}} \rightarrow 2^{\mathbb{R}^{n}}$ is Lipschitz, has compact values and $X \times$ $W_{1} \times W_{2} \subseteq \operatorname{Int}(\operatorname{dom}(F))$. The map ${ }^{2} h: \mathbb{R}^{n} \rightarrow \mathbb{R}^{p}$ satisfies $X \subseteq \operatorname{Int}(\operatorname{dom}(h))$ and $h(X) \subseteq Y$.

Definition 7 Consider the sets $S_{W_{1}} \subseteq \mathbb{R}^{m_{1}}, S_{W_{2}} \subseteq$ $\mathbb{R}^{m_{2}}, S_{X} \subseteq \mathbb{R}^{n}$ and $S_{Y} \subseteq \mathbb{R}^{p} \cdot A$ contract $\mathcal{C}=$ $\left(A_{W_{1}}, A_{W_{2}}, G_{X}, G_{Y}\right)$ is an invariance contract with respect to $\left(S_{W_{1}}, S_{W_{2}}, S_{X}, S_{Y}\right)$ if the set of assumptions and guarantees are described as follows:

- $A_{W_{i}}=\left\{w_{i}: E \rightarrow \mathbb{R}^{m_{i}} \in M\left(\mathbb{R}^{m_{i}}\right) \mid \forall t \in E, w_{i}(t) \in\right.$ $\left.S_{W_{i}}\right\}, i \in\{1,2\}$;

- $G_{X}=\left\{x: E \rightarrow \mathbb{R}^{n} \in M\left(\mathbb{R}^{n}\right) \mid \forall t \in E, x(t) \in S_{X}\right\}$;

- $G_{Y}=\left\{y: E \rightarrow \mathbb{R}^{p} \in M\left(\mathbb{R}^{p}\right) \mid \forall t \in E, y(t) \in S_{Y}\right\}$;

Consider a network of components $\left\{\Sigma^{i}\right\}_{i \in I}$, compatible for composition with respect to $\mathcal{I}$, where each component has the form of (7). Each component $\Sigma^{i}$ has maps and initial sets $F^{i}, h^{i}, X_{0}^{i}, i \in I$, the composed system $\Gamma=$ $\left\langle\left(\Sigma^{i}\right)_{i \in I}, \mathcal{I}\right\rangle$ can be written under the same form with maps $F, h$ and initial set $X_{0}$ given by:

$$
\begin{aligned}
F\left(x, w_{1}\right) & =\prod_{i \in I} F^{i}\left(x^{i}, w_{1}^{i}, w_{2}^{i}\right), w_{2}^{i}=\prod_{j \in \mathcal{N}(i)}\left\{h^{j}\left(x^{j}\right)\right\} \\
h(x) & =\left(h^{1}\left(x^{1}\right), \ldots, h^{N}\left(x^{N}\right)\right), \\
X_{0} & =\prod_{i \in I} X_{0}^{i} .
\end{aligned}
$$

\footnotetext{
1 Given a set-valued map $F: \mathbb{R}^{q} \rightarrow 2^{\mathbb{R}^{n}}$, the domain of $F$ is $\operatorname{dom}(F)=\left\{z \in \mathbb{R}^{q} \mid F(z) \neq \emptyset\right\} . F$ is said to be locally Lipschitz if for all $z \in \operatorname{Int}(\operatorname{dom}(F))$, there exists a neighborhood $U$ of $z$ and a constant $L \geq 0$ (the Lipschitz constant) such that for every $z_{1}, z_{2} \in U \cap \operatorname{dom}(F)$, $F\left(z_{1}\right) \subseteq F\left(z_{2}\right)+L\left\|z_{1}-z_{2}\right\| \mathbb{B}$. $F$ is said to be Lipschitz if the constant $L$ is independent of $z \in \operatorname{Int}(\operatorname{dom}(F))$. It has compact values if for all $z \in \operatorname{dom}(F), F(z)$ is compact.

${ }^{2}$ Given a map $h: \mathbb{R}^{n} \rightarrow \mathbb{R}^{p}$, the domain of $h$ is denoted $\operatorname{dom}(h)$ and consists of elements $x \in \mathbb{R}^{n}$ such that $h(x)$ is defined.
} 
Note that this representation is consistent with the one given in Definition 4 . We have the following technical result whose proof is given in the appendix.

Claim 2 If $h^{i}$ is Lipschitz and Assumption 2 holds for all $\Sigma^{i}, i \in I$, then Assumption 2 holds for $\Gamma=\left\langle\left(\Sigma^{i}\right)_{i \in I}, \mathcal{I}\right\rangle$;

\subsection{Invariants relative to assume-guarantee contracts}

We give necessary and sufficient conditions for weak satisfaction of assume-guarantee contracts based on the classical characterization of invariant sets for differential inclusions (see e.g. Theorem 5.3.4 in [4]).

Definition 8 Let $K \subseteq \mathbb{R}^{n}$ and $x \in K$, the contingent cone to set $K$ at point $x$, denoted $T_{K}(x)$, is given by:

$$
T_{K}(x)=\left\{z \in \mathbb{R}^{n} \mid \liminf _{h \rightarrow 0^{+}} \frac{d_{K}(x+h z)}{h}=0\right\}
$$

where $d_{K}(y)$ denotes the distance of $y$ to $K$, defined by $d_{K}(y)=\inf _{y^{\prime} \in K}\left\|y-y^{\prime}\right\|$.

Definition 9 Let $\Sigma=\left(W_{1}, W_{2}, X, Y, \mathcal{T}\right)$ be a continuoustime system described by (7). Let $\mathcal{C}=\left(A_{W_{1}}, A_{W_{2}}, G_{X}, G_{Y}\right)$ be an invariance assume-guarantee contract for $\Sigma$ with respect to $\left(S_{W_{1}}, S_{W_{2}}, S_{X}, S_{Y}\right)$, where the sets $S_{W_{1}}, S_{W_{2}}$ are compact. $A$ closed set $K \subseteq X$ is said to be an invariant of $\Sigma$ relative to the contract $\mathcal{C}$ if the following conditions hold:

(i) $X_{0} \subseteq K \subseteq S_{X} \cap h^{-1}\left(S_{Y}\right)$;

(ii) for all $x \in K, F\left(x, S_{W_{1}}, S_{W_{2}}\right) \subseteq T_{K}(x)$.

where the set-valued map is given by: $F\left(\cdot, S_{W_{1}}, S_{W_{2}}\right)=$ $\bigcup_{w_{1} \in S_{W_{1}}} \bigcup_{w_{2} \in S_{W_{2}}} F\left(\cdot, w_{1}, w_{2}\right)$.

We prove that the existence of an invariant of $\Sigma$ relative to a contract $\mathcal{C}$ is equivalent to the weak satisfaction of this contract.

Proposition 4 Let $\Sigma=\left(W_{1}, W_{2}, X, Y, \mathcal{T}\right)$ be a continuous-time system described by (7) such that Assumption 2 holds. Let $\mathcal{C}=\left(A_{W_{1}}, A_{W_{2}}, G_{X}, G_{Y}\right)$ be an invariance assume-guarantee contract for $\Sigma$ with respect to $\left(S_{W_{1}}, S_{W_{2}}, S_{X}, S_{Y}\right)$, where the sets $S_{W_{1}}, S_{W_{2}}$ are compact. Then, $\Sigma \models \mathcal{C}$, if and only if there exists a closed set $K \subseteq X$ that is an invariant of $\Sigma$ relative to the contract $\mathcal{C}$

PROOF. First let us prove that the existence of an invariant of $\Sigma$ relative to a contract $\mathcal{C}$ implies the weak satisfaction of this contract. Let $\left(w_{1}, w_{2}, x, y\right): E \rightarrow W_{1} \times W_{2} \times X \times Y$ in $\mathcal{T}$. Let $t \in E$ and suppose that $w_{i \mid[0, t]} \in A_{W_{i}}, i \in\{1,2\}$. Then, we have for all $s \in[0, t], w_{i}(s) \in S_{W_{i}}$, then for almost all $s \in[0, t], \dot{x}(s) \in F\left(x(s), S_{W_{1}}, S_{W_{2}}\right)$. From Assumption 2, we have $X \subseteq \operatorname{Int}\left(\operatorname{dom}\left(F\left(\cdot, S_{W_{1}}, S_{W_{2}}\right)\right)\right)$ and then $K \subseteq \operatorname{Int}\left(\operatorname{dom}\left(\bar{F}\left(\cdot, S_{W_{1}}, S_{W_{2}}\right)\right)\right)$. Moreover, from the compactness of $S_{W_{i}}, i \in\{1,2\}$, it follows that the set-valued map $F\left(\cdot, S_{W_{1}}, S_{W_{2}}\right)$ is Lipschitz and has compact values. Then, since for all $x \in K$, $F\left(x, S_{W_{1}}, S_{W_{2}}\right) \subseteq T_{K}(x)$, we have by Theorem 5.3.4 in [4] that for all $s \in[0, t], x(s) \in K \subseteq S_{X}$ and then for all $s \in[0, t], y(s)=h(x(s)) \in h(K) \subseteq S_{Y}$. Then, $x_{\mid[0, t]} \in G_{X}$ and $y_{\mid[0, t]} \in G_{Y}$. Hence, $\Sigma \models \mathcal{C}$.

We now deal with the second implication. Let us assume that $\Sigma \models \mathcal{C}$. Then for any trajectory $\left(w_{1}, w_{2}, x, y\right): E \rightarrow$ $W_{1} \times W_{2} \times X \times Y$ of the system $\Sigma$. We have for all $t \in E$, if for all $s \in[0, t], w_{1}(s) \in S_{W_{1}}$ and $w_{2}(s) \in S_{W_{1}}$, then for all $s \in[0, t], x(s) \in S_{X}$ and $y(s) \in S_{Y}$. Let us prove the existence of a non empty set $K \subseteq X$ satisfying the conditions of Definition 9. Let us define

$\hat{K}=\left\{p \in X \mid \exists\left(w_{1}, w_{2}, x, y\right): E \rightarrow S_{W_{1}} \times S_{W_{2}} \times X \times Y \in \mathcal{T}\right.$ with $x(0) \in X_{0}$ and $\exists t \in E$ with $x(t)=p$.

The set $\hat{K}$ is the set of reachable states for the differential inclusion (7) initialized in $X_{0}$, where the external and internal inputs belongs to $S_{W_{1}}$ and $S_{W_{2}}$, respectively. From the satisfaction of the contract, we have that $X_{0} \subseteq$ $\hat{K} \subseteq S_{X} \cap h^{-1}\left(S_{Y}\right)$. Let $\left(w_{1}^{\prime}, w_{2}^{\prime}\right) \in S_{W_{1}} \times S_{W_{2}}$ and let us prove that $\hat{K}$ is an invariant for the differential inclusion

$$
\dot{x}(t) \in F\left(x(t), w_{1}^{\prime}, w_{2}^{\prime}\right) \text {. }
$$

Let $z^{0} \in \hat{K}$, and let $z: E^{\prime} \rightarrow X$ be a solution of (10) with $z(0)=z^{0}$. Since $z^{0} \in \hat{K}$, we have the existence of a trajectory $\sigma=\left(w_{1}, w_{2}, x, y\right):[0, s] \rightarrow S_{W_{1}} \times S_{W_{2}} \times X \times$ $Y$ of the system $\Sigma$ described in (7) such that $x(0) \in X_{0}$ and $x(s)=z^{0}$ and for which the external and internal inputs belong to $S_{W_{1}}$ and $S_{W_{2}}$, respectively. Let the time domain $E_{c}$ defined as follows:

$$
\left\{\begin{aligned}
E^{c} & =[0, a+s] \text { if } E^{\prime}=[0, a] \\
& =[0, a+s) \text { if } E^{\prime}=[0, a) \\
& =\mathbb{R}_{0}^{+} \text {if } E^{\prime}=\mathbb{R}_{0}^{+}
\end{aligned}\right.
$$

and let the trajectory $\sigma^{c}=\left(w_{1}^{c}, w_{2}^{c}, x^{c}, y^{c}\right): E^{c} \rightarrow$ $S_{W_{1}} \times S_{W_{2}} \times X \times Y$ of the system $\Sigma$ defined as follows: for all $t \in[0, s], \sigma(t)=\sigma^{c}(t)$ and for all $t \in E^{c} \backslash[0, s]$ we have, $x^{c}(t)=z(t-s), y^{c}(t)=h\left(x^{c}(t)\right), w_{1}^{c}(t)=w_{1}^{\prime}$ and $w_{2}^{c}(t)=w_{2}^{\prime}$. From construction of $\hat{K}$, we have that $x(t) \in \hat{K}$ for all $t \in E^{c}$. Hence, for all $t \in E^{\prime}, z\left(t^{\prime}\right)=$ $x\left(t^{\prime}+s\right) \in \hat{K}$, where $t^{\prime}+s \in E^{c}$. Hence, $\hat{K}$ is an invariant for the differential inclusion (10). Let us now prove that $K=\operatorname{cl}(\hat{K})$ is also an invariant for $(10)$. Let $v^{0} \in K$, and let us assume the existence of $v: E \rightarrow X$ solution to (10) with $v(0)=v^{0}$ and $s \in E$ such that $v(s) \in \bar{K}$. Since, $\bar{K}$ is an open set, we have the existence of $\varepsilon>0$ such that 
$\mathcal{R}_{\varepsilon}(v(s)) \subseteq \bar{K}$. Then, using the continuity of solutions of (10) in initial conditions (see Corollary 5.3.3 in [4]), we have the existence of $\eta>0$ and $x^{0} \in \hat{K}$ such that $x^{0}=x(0) \in \mathcal{R}_{\eta}\left(v^{0}\right)$ and $x(s) \in \mathcal{R}_{\varepsilon}(v(s)) \in \bar{K}$, which contradicts the invariance of $\hat{K}$. Hence, $K=\operatorname{cl}(\hat{K})$ is an invariant for the differential inclusion (10).

For $\left(w_{1}^{\prime}, w_{2}^{\prime}\right) \in S_{W_{1}} \times S_{W_{2}}$, we have that $K$ is closed. Moreover by Assumption 2, $F$ and thus $F\left(\cdot, w_{1}^{\prime}, w_{2}^{\prime}\right)$ is Lipschitz and has compact values. Moreover, $X \times W_{1} \times$ $W_{2} \subseteq \operatorname{Int}(\operatorname{dom}(F))$ and thus $X \subseteq \operatorname{Int}\left(F\left(\cdot, w_{1}^{\prime}, w_{2}^{\prime}\right)\right)$, which in turn implies that $K \subseteq \operatorname{Int}\left(F\left(\cdot, w_{1}^{\prime}, w_{2}^{\prime}\right)\right)$. Then, from Theorem 5.3.4 in [4], we have

$$
\forall x \in K, F\left(x, w_{1}^{\prime}, w_{2}^{\prime}\right) \subseteq T_{K}(x) .
$$

Since equation (11) is verified for all $\left(w_{1}^{\prime}, w_{2}^{\prime}\right) \in S_{W_{1}} \times$ $S_{W_{2}}$, we have

$\forall x \in K, F\left(\cdot, S_{W_{1}}, S_{W_{2}}\right)=\bigcup_{w_{1}^{\prime} \in S_{W_{1}}} \bigcup_{w_{2}^{\prime} \in S_{W_{2}}} F\left(\cdot, w_{1}^{\prime}, w_{2}^{\prime}\right) \subseteq T_{K}(x)$ Then, $K$ is an invariant of the system $\Sigma$ relative to the contract $\mathcal{C}$.

It is noteworthy to mention that in view of Proposition 4, the Lipschitzness property of the system $\Sigma$ is needed only on a neighbourhood of the set of interest given by $S_{X} \times S_{W_{1}} \times S_{W_{2}}$.

\subsection{Composition of invariants}

We now provide results allowing us to reason about interconnected systems based on invariants of their components.

Theorem 3 Consider a network of continuous-time components $\left\{\Sigma^{i}\right\}_{i \in I}$ compatible for composition with respect to $\mathcal{I}$, where the components $\Sigma_{i}, i \in I$, have the form of (7) and satisfies Assumption 2. Each component $\Sigma^{i}$ have maps and initial sets $F^{i}, h^{i}, X_{0}^{i}, i \in I$, where $h^{i}$ is Lipschitz. Let $\mathcal{C}^{i}=\left(A_{W_{1}^{i}}, A_{W_{2}^{i}}, G_{X^{i}}, G_{Y^{i}}\right)$ be an invariance assume-guarantee contract for $\Sigma^{i}$ with respect to $\left(S_{W_{1}^{i}}, S_{W_{2}^{i}}, S_{X^{i}}, S_{Y^{i}}\right)$, where the sets $S_{W_{1}^{i}}, S_{W_{2}^{i}}$ are compact. Let $\mathcal{C}=\left(\prod_{i \in I} A_{W_{1}^{i}}, \prod_{i \in I} G_{X^{i}}, \prod_{i \in I} G_{Y^{i}}\right)$ be a contract for the composed system $\Gamma=\left\langle\left(\Sigma^{i}\right)_{i \in I}, \mathcal{I}\right\rangle$ and assume the following:

(i) for all $i \in I, \Sigma^{i}=\mathcal{C}^{i}$;

(ii) for all $i \in I, \prod_{j \in \mathcal{N}(i)} S_{Y^{j}} \subseteq S_{W_{2}^{i}}$.

then $\Gamma \models \mathcal{C}$.

PROOF. First, since $h^{i}$ is Lipschitz and Assumption 2 holds for all $\Sigma^{i}, i \in I$, then from Claim 2 we have that
Assumption 2 holds for $\Gamma=\left\langle\left(\Sigma^{i}\right)_{i \in I}, \mathcal{I}\right\rangle$. Hence, using the equivalence between the invariance relative to contracts and the weak satisfaction of contracts from Proposition 4, we have the existence of a closed set $K^{i}$ that is invariant for $\Sigma^{i}$ relative to the contract $\mathcal{C}^{i}$.

To complete the proof, let us show that the set $K=$ $\prod_{i \in I} K^{i}$ is an invariant of $\Gamma$ relative to the contract $\mathcal{C}$. Let $i \in I$ and consider a trajectory $x^{i}: \mathbb{R}_{0}^{+} \rightarrow X^{i}$ with $x^{i}(0) \in K^{i}$ and satisfying $\dot{x}^{i}(t) \in F^{i}\left(x^{i}, S_{W_{1}^{i}}, S_{W_{2}^{i}}\right)$, for all $t \in \mathbb{R}_{0}^{+}$. Using the fact that $K^{i}$ invariant of $\Sigma^{i}$ relative to the contract $\mathcal{C}^{i}$ and from Proposition 4 we have that $x^{i}(t) \in K^{i}$, for all $t \in \mathbb{R}_{0}^{+}$.

Consider the map $G: X \rightarrow 2^{X}$ defined for $x \in X$ by:

$$
G(x)=\prod_{i \in I} F^{i}\left(x^{i}, S_{W_{1}^{i}}, S_{W_{2}^{i}}\right)
$$

Let a trajectory $x: \mathbb{R}_{0}^{+} \rightarrow X$ with $x(0) \in K$ and satisfying $\dot{x}(t) \in G(x(t))$ for all $t \in \mathbb{R}_{0}^{+}$. We have from above that $x(t) \in K$, for all $t \in \mathbb{R}_{0}^{+}$. Moreover, using the fact that $G$ is Lipschitz, has compact values, $X \subseteq \operatorname{Int}(\operatorname{dom}(G))$ and $K$ is a closed set, we have by Theorem 5.3.4 in [4] that $G(x) \subseteq T_{K}(x)$ for all $x \in K$.

Let us now consider the system described by the following differential inclusion:

$$
\dot{x}(t) \in F\left(x(t), S_{W_{1}}\right) .
$$

Where $x(t)=\left(x_{1}(t), \ldots, x_{N}(t)\right)$ and $S_{W_{1}}=\prod_{i \in I} S_{W_{1}^{i}}$.

Let $x \in K$, then for all $i \in I$, we have that

$$
\begin{aligned}
F^{i}\left(x^{i}, S_{W_{1}^{i}}, w_{2}^{i}\right) & =F^{i}\left(x^{i}, S_{W_{1}^{i}}, \prod_{j \in \mathcal{N}(i)}\left\{h^{j}\left(x^{j}\right)\right\}\right) \\
& \subseteq F^{i}\left(x^{i}, S_{W_{1}^{i}}, \prod_{j \in \mathcal{N}(i)}\left\{h^{j}\left(K^{j}\right)\right\}\right) \\
& \subseteq F^{i}\left(x^{i}, S_{W_{1}^{i}}, \prod_{j \in \mathcal{N}(i)}\left\{S_{Y^{j}}\right\}\right) \\
& \subseteq F^{i}\left(x^{i}, S_{W_{1}^{i}}, S_{W_{2}^{i}}\right) .
\end{aligned}
$$

Where the first equality comes from the definition of an interconnection relation, the second inclusion comes from (i) and the last inclusion comes from (ii). Then, we have that $F\left(x, S_{W_{1}}\right) \subseteq G(x) \subseteq T_{K}(x)$, for all $x \in K$. Finally, we have $X_{0}=\prod_{i \in I} \bar{X}_{0}^{i} \subseteq \prod_{i \in I} K^{i}=K$, $K=\prod_{i \in I} K^{i} \subseteq \prod_{i \in I} G_{X^{i}}$, and $K=\prod_{i \in I} K^{i} \subseteq$ $\prod_{i \in I}\left(h^{i}\right)^{-1}\left(G_{Y^{i}}\right)=h^{-1}\left(G_{Y}\right)$. Hence, $K$ is an invariant of $\Gamma$ relative to the contract $\mathcal{C}$. Then from Proposition 4 and Claim 2, we get $\Gamma \models \mathcal{C}$.

We show an example to illustrate the application of the previous theorem. 
Example 11 Consider systems $\Sigma^{i}=\left(W_{1}^{i}, W_{2}^{i}, X_{i}, Y_{i}, \mathcal{T}_{i}\right)$ $i=1,2$ where $W_{1}^{i}=W_{2}^{i}=X^{i}=Y^{i}=\mathbb{R}$. A trajectory of $\Sigma^{i}$ is a triple $\left(w_{1}^{i}, w_{2}^{i}, x^{i}, y^{i}\right): E \rightarrow W_{1}^{i} \times W_{2}^{i} \times X^{i} \times Y^{i}$ in $\mathcal{T}_{i}$ where $E=\mathbb{R}_{0}^{+}, w_{1}^{i}$ and $w_{2}^{i}$ are locally measurable, $x^{i}$ and $y^{i}$ are absolutely continuous and continuous, respectively, and satisfy for almost all $t \in E$ :

$$
\left\{\begin{aligned}
\dot{x}^{i}(t) & =f^{i}\left(x^{i}(t), w_{1}^{i}(t), w_{2}^{i}(t)\right) \\
& =-a_{i} x^{i}(t)+a_{i} w_{2}^{i}(t)+w_{1}^{i}(t) \\
y^{i}(t) & =h^{i}(x(t))=x^{i}(t) .
\end{aligned}\right.
$$

where $x_{i}(0) \in\left[0, b_{i}\right]$ with $a_{i}, b_{i} \in \mathbb{R}_{0}^{+}$, let $b=\max \left(b_{1}, b_{2}\right)$. It can be seen that $h_{i}$ is Lipschitz and that Assumption 2 holds for $\Sigma_{i}$. Let the interconnection relation $\mathcal{I}=$ $\{(1,2),(2,1)\}$. It is clear that $\left\{\Sigma^{i}\right\}_{i \in I}$ is compatible for composition with respect to $\mathcal{I}$. Let the invariance contract $\mathcal{C}^{i}=\left(A_{W_{1}^{i}}, A_{W_{2}^{i}}, G_{X^{i}}, G_{Y^{i}}\right)$ for the system $\Sigma^{i}$ with respect to $\left(S_{W_{1}^{i}}, S_{W_{2}^{i}}, S_{X^{i}}, S_{Y^{i}}\right)$, with $S_{W_{1}^{i}}=\{0\}, S_{W_{2}^{i}}=$ $S_{X^{i}}=S_{Y^{i}}=[0, b]$. We can easily check that for all $x^{i} \in[0, b], f_{i}\left(x^{i},[0, b],\{0\}\right) \subseteq T_{[0, b]}\left(x^{i}\right)$, since

$$
T_{[0, b]}\left(x^{i}\right)=\left\{\begin{array}{l}
\mathbb{R}^{+} \quad \text { if } x^{i}=0, \\
\mathbb{R}^{-} \text {if } x^{i}=b, \\
\mathbb{R} \quad \text { if } x^{i} \in(0, b)
\end{array}\right.
$$

Then $[0, b]$ is an invariant of the system $\Sigma_{i}$, relative to the contract $\mathcal{C}^{i}$. By Theorem 3, $[0, b]^{2}$ is an invariant of the composed system $\Gamma=\left\langle\left(\Sigma^{i}\right)_{i \in I}, \mathcal{I}\right\rangle$ relative to the composed contract $\mathcal{C}$.

\section{$7 \quad$ Small gain results}

In this part, we show how the proposed framework can recover different versions of the classical small gain theorem as a particular case. Indeed, we show how the framework allows to recover the classical BIBO stability result [12]. Moreover, we construct a new small-gain result for the concept of growth bound [2]. To the best of our knowledge, this result is new and have not been investigated before in the literature. We suppose for the sake of simplicity that for each system $\Sigma=\left(W_{1}, W_{2}, X, Y, \mathcal{T}\right)$, we have $W_{1}=\{0\}, X=Y=W_{2}=\mathbb{R}^{n}$ and for all $\left(w_{1}, w_{2}, x, y\right): \mathbb{R}_{0}^{+} \rightarrow W_{1} \times W_{2} \times W \times X \times Y$ in $\mathcal{T}$, $x(t)=y(t)$, for all $t \in \mathbb{R}_{0}^{+}$.

\subsection{BIBO stability}

Given a system $\Sigma$ satisfying a BIBO stability condition [12], we show that if the gain of the system is lower than 1 then the feedback ${ }^{3}$ composed system is bounded

\footnotetext{
$\overline{3}$ Given a system $\Sigma^{1}$ and a set of vertices $I=\{1\}$, the feedback composition of the system $\Sigma^{1}$ is the composition with an interconnection relation $\mathcal{I}=\{(1,1)\}$.
}

for all the time domain.

Theorem 4 Consider a component $\Sigma^{1}=\left(\{0\}, W_{2}, X, Y, \mathcal{T}\right)$, $I=\{1\}$ and let the interconnection relation $\mathcal{I}=\{(1,1)\}$ such that $\left\{\Sigma^{i}\right\}_{i \in I}$ is compatible for composition with respect to $\mathcal{I}$. Let $\gamma<1$ and $\beta \in \mathbb{R}_{0}^{+}$such that for any trajectory $\left(0, w_{2}, x, y\right): \mathbb{R}_{0}^{+} \rightarrow W_{1} \times W_{2} \times X \times Y$ in $\mathcal{T}$, $x: \mathbb{R}_{0}^{+} \rightarrow X$ is continuous, $\|x(0)\| \leq \frac{\beta}{1-\gamma}$ and for all $t \in \mathbb{R}_{0}^{+}$we have:

$$
\left\|x_{\mid[0, t]}\right\|_{\infty} \leq \gamma\left\|w_{2 \mid[0, t]}\right\|_{\infty}+\beta
$$

Then for any trajectory $(0, x, y): \mathbb{R}_{0}^{+} \rightarrow\{0\} \times X \times Y$ of the composed system $\Gamma=\left\langle\left(\Sigma^{i}\right)_{i \in I}, \mathcal{I}\right\rangle$, we have for all $t \in \mathbb{R}_{0}^{+}:\left\|x_{\mid[0, t]}\right\|_{\infty} \leq \frac{\beta}{1-\gamma}$.

PROOF. We first start by constructing a suitable contract for the system $\Sigma^{1}$. Let the map $a: \mathbb{R}_{0}^{+} \rightarrow \mathbb{R}_{0}^{+}$, a parameter $\varepsilon>0$ and a parametrized contract $\mathcal{C}(\varepsilon)=$ $\left(A_{W_{1}}^{\varepsilon}, A_{W_{2}}^{\varepsilon}, G_{X}^{\varepsilon}, G_{Y}^{\varepsilon}\right)$ for $\Sigma$, where:

- $A_{W_{1}}^{\varepsilon}=\left\{w_{1}: \mathbb{R}_{0}^{+} \rightarrow W_{1} \in M\left(W_{1}\right) \mid \forall t \in \mathbb{R}_{0}^{+}, w_{1}(t)=\right.$ $0\}$;

- $A_{W_{2}}^{\varepsilon}=\left\{w_{2}: \mathbb{R}_{0}^{+} \rightarrow W_{2} \in M\left(W_{2}\right) \mid \forall t \in \mathbb{R}_{0}^{+},\left\|w_{2 \mid[0, t]}\right\|_{\infty} \leq\right.$ $a(\varepsilon)\}$

- $G_{X}^{\varepsilon}=G_{Y}^{\varepsilon}=\left\{x: \mathbb{R}_{0}^{+} \rightarrow X \in M(X) \mid \forall t \in\right.$ $\left.\mathbb{R}_{0}^{+},\left\|x_{\mid[0, t]}\right\|_{\infty} \leq \gamma a(\varepsilon)+\beta\right\}$.

Let us choose $a(\varepsilon)=\frac{\beta+\varepsilon}{1-\gamma}$, where $\varepsilon>0$. We have that

$$
\|x(0)\| \leq \frac{\beta}{1-\gamma} \leq \gamma a(\varepsilon)+\beta=\frac{\beta+\gamma \varepsilon}{1-\gamma}
$$

for any $\varepsilon>0$. Hence, $x_{\mid[0,0]} \in G_{X}^{\varepsilon}$. We also have from (14) that $\Sigma^{1} \models \mathcal{C}(\varepsilon)$, and for all trajectories $\left(w_{1}, w_{2}, x, y\right) \in \mathcal{T}, x: \mathbb{R}_{0}^{+} \rightarrow X$ is continuous. Then, from Proposition 2, we have that $\Sigma^{1} \|_{s} \mathcal{C}^{\prime}(\varepsilon)$ for any $\varepsilon>0$, where $\mathcal{C}^{\prime}(\varepsilon)=\left(A_{W_{1}}^{\varepsilon}, A_{W_{2}}^{\varepsilon}, G_{X}^{\varepsilon}, \mathcal{B}_{\varepsilon}\left(G_{Y}^{\varepsilon}\right) \cap M(Y)\right)$. Now, using the fact that $\gamma a(\varepsilon)+\beta-a(\varepsilon)=-\varepsilon<0$, we have that $\mathcal{B}_{\varepsilon}\left(G_{Y}^{\varepsilon}\right) \cap M(Y) \subseteq A_{W_{2}}^{\varepsilon}$. Moreover, from continuity of $x: \mathbb{R}_{0}^{+} \rightarrow X$ Assumption 1 is satisfied. Then from Theorem 2, the composed system $\Gamma=\left\langle\left(\Sigma^{i}\right)_{i \in I}, \mathcal{I}\right\rangle$ satisfies the composed contract $\mathcal{C}_{\Gamma}^{\varepsilon}=\left(A_{W_{1}}^{\varepsilon}, G_{X}^{\varepsilon}, \mathcal{B}_{\varepsilon}\left(G_{Y}^{\varepsilon}\right) \cap M(Y)\right)$. Then, we have for all $t \in \mathbb{R}_{0}^{+}:\left\|x_{\mid[0, t]}\right\|_{\infty} \leq \gamma a(\varepsilon)+\beta=\frac{\beta+\gamma \varepsilon}{1-\gamma}$.

Since the last inequality is verified for all $\varepsilon>0$ we have for all $t \in \mathbb{R}_{0}^{+},\left\|x_{\mid[0, t]}\right\|_{\infty} \leq \frac{\beta}{1-\gamma}$.

\subsection{Growth bound}

The notion of growth bound allows us to analyse the growth or contraction properties of a system; 
particularly, it coincides with forward completeness (see Corollary 2.3 in [2]) for finite-dimensional systems described by nonlinear differential equations $\dot{x}(t)=F\left(x(t), w_{1}(t), w_{2}(t)\right)$ and with a locally Lipschitz map $F$. Given a continuous-time system with a given growth bound, in the following we show how to characterize the growth bound of the feedback composed system.

Theorem 5 Consider a component $\Sigma^{1}=\left(\{0\}, W_{2}, X, Y, \mathcal{T}\right)$ $I=\{1\}$ and let $\mathcal{I}=\{(1,1)\}$ be the interconnection relation such that $\left\{\Sigma^{i}\right\}_{i \in I}$ is compatible for composition with respect to $\mathcal{I}$. Let $\gamma_{1}, \gamma_{2}, \gamma_{3}$ be class $\mathcal{K}$ maps and constants $(c, \rho) \in \mathbb{R} \times[0,1)$, where $\gamma_{3}(\cdot)<\rho \operatorname{Id}(\cdot)^{4}$ and such that for any trajectory $\left(0, w_{2}, x, y\right): \mathbb{R}_{0}^{+} \rightarrow W_{1} \times W_{2} \times X \times Y$ in $\mathcal{T}, x: \mathbb{R}_{0}^{+} \rightarrow X$ is continuous, $\|x(0)\| \leq \gamma_{2}(\|x(0)\|)+c$ and for all $t \in \mathbb{R}_{0}^{+}$we have:

$$
\|x(t)\| \leq \gamma_{1}(t)+\gamma_{2}(\|x(0)\|)+\gamma_{3}\left(\left\|w_{2 \mid[0, t]}\right\|_{\infty}\right)+c .
$$

Then there exist $\mathcal{K}$ functions $\alpha_{1}, \alpha_{2}$ and $c^{\prime} \in \mathbb{R}$ such that for any trajectory $(0, x, y): \mathbb{R}_{0}^{+} \rightarrow\{0\} \times X \times Y$ of the composed system $\Gamma=\left\langle\left(\Sigma^{i}\right)_{i \in I}, \mathcal{I}\right\rangle$ we have for all $t \in \mathbb{R}_{0}^{+}$, $\|x(t)\| \leq \alpha_{1}(t)+\alpha_{2}(\|x(0)\|)+c^{\prime}$, where $\alpha_{1}=\frac{2}{1-\rho} \gamma_{1}$, $\alpha_{2}=\frac{2}{1-\rho} \gamma_{2}$, and $c^{\prime}=\frac{2 c}{1-\rho}$.

PROOF. We first define the system $\Sigma_{x(0)}^{1}=\left(\{0\}, W_{2}, X\right.$, $\left.Y, \mathcal{T}_{x(0)}\right)$ where $\left(0, w_{2}, x, y\right): \mathbb{R}_{0}^{+} \rightarrow\{0\} \times W_{2} \times X \times Y \in$ $\mathcal{T}_{x(0)}$ is a trajectory of the system $\Sigma_{x(0)}^{1}$ is and only if it is a trajectory of the system $\Sigma^{1}$ initialized in $x(0) \in X$.

We start by constructing a suitable contract for the system $\Sigma_{x(0)}^{1}$. Let the map $a: \mathbb{R}_{0}^{+} \times \mathbb{R}_{0}^{+} \rightarrow \mathbb{R}_{0}^{+}$. A parameter $\varepsilon>0$ and a parametrized contract $\mathcal{C}(\varepsilon)=$ $\left(A_{W_{1}}^{\varepsilon}, A_{W_{2}}^{\varepsilon}, G_{X}^{\varepsilon}, G_{Y}^{\varepsilon}\right)$ for $\Sigma^{1}$, where:

- $A_{W_{1}}^{\varepsilon}=\left\{w_{1}: \mathbb{R}_{0}^{+} \rightarrow W_{1} \in M\left(W_{1}\right) \mid \forall t \in \mathbb{R}_{0}^{+}, w_{1}(t)=\right.$ $0\}$;

- $A_{W_{2}}^{\varepsilon}=\left\{w_{2}: \mathbb{R}_{0}^{+} \rightarrow W_{2} \in M\left(W_{2}\right) \mid \forall t \in \mathbb{R}_{0}^{+},\left\|w_{2}(t)\right\| \leq\right.$ $a(t, \varepsilon)\}$

- $G_{X}^{\varepsilon}=G_{Y}^{\varepsilon}=\left\{x: \mathbb{R}_{0}^{+} \rightarrow X \in M(X) \mid \forall t \in\right.$ $\left.\mathbb{R}_{0}^{+},\|x(t)\| \leq \gamma_{1}(t)+\gamma_{2}(\|x(0)\|)+\gamma_{3}(\|a(t, \varepsilon)\|)+c\right\}$.

Let us choose the map $a: \mathbb{R}_{0}^{+} \times \mathbb{R}_{0}^{+} \rightarrow \mathbb{R}_{0}^{+}$satisfying

$$
a(t, \varepsilon)=(1-\rho)^{-1}\left(\gamma_{1}(t)+\gamma_{2}(\|x(0)\|)+c+\varepsilon\right)
$$

where $\varepsilon>0$. First, since $\rho<1$, we have that $(1-\rho)$ Id is a class $\mathcal{K}$ map, which implies from Lemma 4.2 in [21]) that $(1-\rho)^{-1} \mathrm{Id}$ is class $\mathcal{K}$. Moreover, since $\gamma_{1}$ is class $\mathcal{K}$ we have for all $t \in \mathbb{R}_{0}^{+}$,

$$
\left\|a_{\mid[0, t]}(\cdot, \varepsilon)\right\|_{\infty}=\|a(t, \varepsilon)\|
$$

\footnotetext{
$\overline{4}$ i.e, $\gamma_{3}(r)<\rho r$ for all $r>0$
}

Let us now prove that $\Sigma^{1} \models \mathcal{C}(\varepsilon)$. Let $t \in \mathbb{R}_{0}^{+}$and assume that $\left\|w_{2}(s)\right\| \leq a(s, \varepsilon)$ for all $s \in[0, t]$. We have from (15) that for all $s \in[0, t]$

$$
\begin{aligned}
\|x(s)\| & \leq \gamma_{1}(s)+\gamma_{2}(\|x(0)\|)+\gamma_{3}\left(\left\|w_{2 \mid[0, s]}\right\|_{\infty}\right)+c \\
& \leq \gamma_{1}(s)+\gamma_{2}(\|x(0)\|)+\gamma_{3}\left(\left\|a_{\mid[0, s]}(\cdot, \varepsilon)\right\|_{\infty}\right)+c \\
& \leq \gamma_{1}(s)+\gamma_{2}(\|x(0)\|)+\gamma_{3}(\|a(s, \varepsilon)\|)+c
\end{aligned}
$$

where the last inequality comes from (16). Hence, $\Sigma^{1} \models \mathcal{C}(\varepsilon)$. Moreover we have that for all trajectories $\left(w_{1}, w_{2}, x, y\right) \in \mathcal{T}, x: \mathbb{R}_{0}^{+} \rightarrow X$ is continuous and using the fact that $\|x(0)\| \leq \gamma_{2}(\|x(0)\|)+c$, we have that $x_{\mid[0,0]} \in G_{X}^{\varepsilon}$, for all $\varepsilon>0$. Then, from Proposition 2, we have that $\Sigma^{1} \models{ }_{s} \mathcal{C}^{\prime}(\varepsilon)$ for any $\varepsilon>0$, where $\mathcal{C}^{\prime}(\varepsilon)=\left(A_{W_{1}}^{\varepsilon}, A_{W_{2}}^{\varepsilon}, G_{X}^{\varepsilon}, \mathcal{B}_{\varepsilon}\left(G_{Y}^{\varepsilon}\right) \cap M(Y)\right)$. Now, using the fact that $\gamma_{3}<\rho$ Id we have

$$
\begin{aligned}
\gamma_{1}(t)+\gamma_{2}(\|x(0)\|)+ & \gamma_{3}(a(t, \varepsilon))+c-a(t, \varepsilon) \leq \gamma_{1}(t)+ \\
& \gamma_{2}(\|x(0)\|)+\rho a(t, \varepsilon)+c-a(t, \varepsilon) \\
= & -\varepsilon<0
\end{aligned}
$$

which implies that $\mathcal{B}_{\varepsilon}\left(G_{Y}^{\varepsilon}\right) \cap M(Y) \subseteq A_{W_{2}}^{\varepsilon}$. Moreover, from continuity of $x: \mathbb{R}_{0}^{+} \rightarrow X$, Assumption 1 is satisfied. Then from Theorem 2 , the composed system $\Gamma=\left\langle\left(\Sigma^{i}\right)_{i \in I}, \mathcal{I}\right\rangle$ satisfies the composed contract $\mathcal{C}_{\Gamma}^{\varepsilon}=\left(A_{W_{1}}^{\varepsilon}, G_{X}^{\varepsilon}, \mathcal{B}_{\varepsilon}\left(G_{Y}^{\varepsilon}\right) \cap M(Y)\right)$. Then, we have for all $t \in \mathbb{R}_{0}^{+}$:

$$
\begin{aligned}
\|x(t)\| & \leq \gamma_{1}(t)+\gamma_{2}(\|x(0)\|)+\gamma_{3}(a(t, \varepsilon))+c \\
& \leq a(t, \varepsilon)=(1-\rho)^{-1}\left(\gamma_{1}(t)+\gamma_{2}(\|x(0)\|)+c+\varepsilon\right) .
\end{aligned}
$$

The last inequality is verified for all $\varepsilon>0$, which implies from the continuity of $(1-\rho)^{-1}$ Id that for all $t \in \mathbb{R}_{0}^{+}$:

$$
\begin{aligned}
\|x(t)\| & \leq(1-\rho)^{-1}\left(\gamma_{1}(t)+\gamma_{2}(\|x(0)\|)+c\right) \\
& \leq \frac{2}{1-\rho} \gamma_{1}(t)+\frac{2}{1-\rho} \gamma_{2}(\|x(0)\|) \\
& +\frac{2 c}{1-\rho}
\end{aligned}
$$

where the last inequality comes from the fact that $(1-$ $\rho)$ Id is a class $\mathcal{K}$ and the weak triangular inequality [19]. By choosing $\alpha_{1}=\frac{2}{1-\rho} \gamma_{1}, \alpha_{2}=\frac{2}{1-\rho} \gamma_{2}$, and $c^{\prime}=\frac{2 c}{1-\rho}$ where $\alpha_{1}$ and $\alpha_{2}$ are class $\mathcal{K}$ (see Lemma 4.2 in [21]), we have for all $t \in \mathbb{R}_{0}^{+},\|x(t)\| \leq \alpha_{1}(t)+\alpha_{2}(\|x(0)\|)+c^{\prime}$.

We can mention that for finite-dimensional systems, described by nonlinear differential equations and with a locally Lipschitz map $F$, the previous result states that if a system is forward complete with a gain $\gamma_{3}$ lower than identity, then the feedback composed system is forward complete.

Remark 8 Let us mention that using the same approach, and similar to the work of [11], one can generalize 


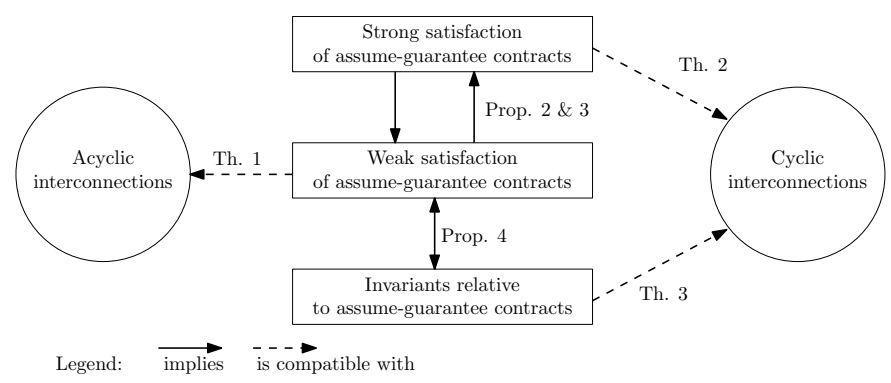

Fig. 5. Summary of main results in the paper

different small-gain results to different interconnection structures.

\section{Conclusion}

In this paper, we proposed a contract based approach for verifying compositionally properties of continuoustime interconnected systems. The main notions considered in the paper and their relationships are sketched in Figure 5. The main contributions are summarized below. We introduced a notion of assume-guarantee contracts equipped with a weak and a strong semantics. We showed that weak semantics are sufficient to deal with acyclic interconnections (Theorem 1), strong semantics are required to reason on cyclic interconnections (Theorem 2 and Example 6) and that strong semantics of a contract can sometimes be obtained from weak ones (Propositions 2 and 3).

We then developed specific results for systems described by differential inclusions and invariance assumeguarantee contracts. We showed that sufficient and necessary conditions for weak satisfaction of contracts can be given using invariant sets (Proposition 4) and that invariants are compatible with cyclic interconnections (Theorem 3). Finally, we have shown how the proposed assume-guarantee framework can recast different versions of the small-gain theorem as a particular case (Theorems 4 and 5).

\section{Appendix}

\section{Proof of Claim 2}

Consider a network of components $\left\{\Sigma^{i}\right\}_{i \in I}$, compatible for composition w.r.t. $\mathcal{I}$, where each component has the form of (7). Each component $\Sigma^{i}$ has maps and initial sets $F^{i}, h^{i}, X_{0}^{i}, i \in I$, and the composed system $\Gamma=$ $\left\langle\left(\Sigma^{i}\right)_{i \in I}, \mathcal{I}\right\rangle$ can be written as in (8). Assume that $h^{i}$ is Lipschitz and Assumption 2 holds for all $\Sigma_{i}, i \in I$, and let us show that $\Gamma=\left\langle\left(\Sigma^{i}\right)_{i \in I}, \mathcal{I}\right\rangle$ satisfies Assumption 2.

First, using the fact that for all $i \in I, F^{i}$ has compact values and $X^{i} \times W_{1}^{i} \times W_{2}^{i} \subseteq \operatorname{Int}\left(\operatorname{dom}\left(F^{i}\right)\right)$, we have directly from (8) that $F$ has compact values and $X \times$ $W_{1} \subseteq \operatorname{Int}(\operatorname{dom}(F))$.

Similarly, using the fact that for all $i \in I, h^{i}$ satisfies $X^{i} \subseteq \operatorname{Int}\left(\operatorname{dom}\left(h^{i}\right)\right)$ and $h^{i}\left(X^{i}\right) \subseteq Y^{i}$, we directly get from (8) that $h$ satisfies $X \subseteq \operatorname{Int}(\operatorname{dom}(h))$ and $h(X) \subseteq$ $Y$.

Now assume that for all $i \in I, F^{i}$ and $h^{i}$ are Lipchitz, and let us show that the interconnected system $\Gamma=\left\langle\left(\Sigma^{i}\right)_{i \in I}, \mathcal{I}\right\rangle$ is Lipschitz. From Lipschitzness of $F^{i}$, we have the existence of $L^{i}, L_{1}^{i}, L_{2}^{i}>0$ such that for all $\left(x_{a}^{i}, x_{b}^{i}\right) \in X^{i}$, for all $\left(w_{1, a}^{i}, w_{1, b}^{i}\right) \in W_{1}^{i}$ and for all $\left(w_{2, a}^{i}, w_{2, b}^{i}\right) \in W_{2}^{i}$, we have :

$$
\begin{aligned}
F^{i}\left(x_{a}^{i}, w_{1, a}^{i}, w_{2, a}^{i}\right) & \subseteq F^{i}\left(x_{b}^{i}, w_{1, b}^{i}, w_{2, b}^{i}\right)+L^{i}\left\|x_{a}^{i}-x_{b}^{i}\right\| \mathbb{B} \\
& +L_{1}^{i}\left\|w_{1, a}^{i}-w_{1, b}^{i}\right\| \mathbb{B}+L_{2}^{i}\left\|w_{2, a}^{i}-w_{2, b}^{i}\right\| \mathbb{B}
\end{aligned}
$$

where $\|$.$\| is the infinity norm of appropriate dimension$ and $\mathbb{B}$ is the ball centred at the origin with radius 1 of appropriate dimension. Similarly, from Lipschitzness of $h^{i}$, we have the existence of $l^{i}>0$ such that for all $\left(x_{a}^{i}, x_{b}^{i}\right) \in X^{i}$ we have:

$$
\left\|h^{i}\left(x_{a}^{i}\right)-h^{i}\left(x_{a}^{i}\right)\right\| \leq l^{i}\left\|x_{a}^{i}-x_{b}^{i}\right\|
$$

Consider $\left(x_{a}, x_{b}\right) \in X$ and $\left(w_{1, a}, w_{1, b}\right) \in W_{1}$ with $x_{a}=\left(x_{a}^{1}, \ldots, x_{a}^{N}\right), x_{b}=\left(x_{b}^{1}, \ldots, x_{b}^{N}\right), w_{1, a}=$ $\left(w_{1, a}^{1}, \ldots, w_{1, a}^{N}\right)$, and $w_{1, b}=\left(w_{1, b}^{1}, \ldots, w_{1, b}^{N}\right)$. Choosing $L=\max _{i \in I} L^{i}, L_{1}=\max _{i \in I} L_{1}^{i}, L_{2}=\max _{i \in I} L_{2}^{i}$ and $l=\max _{i \in I} l^{i}$, we have the chain of inclusions in (17). Hence, the interconnected system $\Gamma=\left\langle\left(\Sigma^{i}\right)_{i \in I}, \mathcal{I}\right\rangle$ is Lipschitz.

\section{References}

[1] R. Alur, R. Grosu, I. Lee, and O. Sokolsky. Compositional refinement for hierarchical hybrid systems. In International Workshop on Hybrid Systems: Computation and Control, pages 33-48. Springer, 2001.

[2] David Angeli and Eduardo D Sontag. Forward completeness, unboundedness observability, and their lyapunov characterizations. Systems $\&$ Control Letters, 38(45):209-217, 1999 .

[3] M. Arcak, C. Meissen, and A Packard. Networks of dissipative systems: compositional certification of stability, performance, and safety. Springer, 2016.

[4] J.-P. Aubin. Viability theory. Springer Science \& Business Media, 2009.

[5] Christel Baier and Joost-Pieter Katoen. Principles of model checking. MIT press, 2008.

[6] Albert Benveniste, Benoît Caillaud, Dejan Nickovic, Roberto Passerone, Jean-Baptiste Raclet, Philipp Reinkemeier, Alberto Sangiovanni-Vincentelli, Werner Damm, Thomas A 


$$
\begin{aligned}
F\left(x_{a}, w_{1, a}\right) & =\prod_{i \in I} F^{i}\left(x_{a}^{i}, w_{1, a}^{i}, w_{2, a}^{i}\right) \\
& \subseteq \prod_{i \in I}\left(F^{i}\left(x_{b}^{i}, w_{1, b}^{i}, w_{2, b}^{i}\right)+L^{i}\left\|x_{a}^{i}-x_{b}^{i}\right\| \mathbb{B}+L_{1}^{i}\left\|w_{1, a}^{i}-w_{1, b}^{i}\right\| \mathbb{B}+L_{2}^{i}\left\|w_{2, a}^{i}-w_{2, b}^{i}\right\| \mathbb{B}\right) \\
& \subseteq \prod_{i \in I}\left(F^{i}\left(x_{b}^{i}, w_{1, b}^{i}, w_{2, b}^{i}\right)+L\left\|x_{a}^{i}-x_{b}^{i}\right\| \mathbb{B}+L_{1}\left\|w_{1, a}^{i}-w_{1, b}^{i}\right\| \mathbb{B}+L_{2}\left\|w_{2, a}^{i}-w_{2, b}^{i}\right\| \mathbb{B}\right) \\
& \subseteq \prod_{i \in I}\left(F^{i}\left(x_{b}^{i}, w_{1, b}^{i}, w_{2, b}^{i}\right)+L\left\|x_{a}^{i}-x_{b}^{i}\right\| \mathbb{B}+L_{1}\left\|w_{1, a}^{i}-w_{1, b}^{i}\right\| \mathbb{B}+L_{2}\left\|\prod_{j \in \mathcal{N}(i)}\left\{h^{j}\left(x_{a}^{j}\right)\right\}-\prod_{j \in \mathcal{N}(i)}\left\{h^{j}\left(x_{b}^{j}\right)\right\}\right\| \mathbb{B}\right) \\
& \subseteq \prod_{i \in I}\left(F^{i}\left(x_{b}^{i}, w_{1, b}^{i}, w_{2, b}^{i}\right)+L\left\|x_{a}^{i}-x_{b}^{i}\right\| \mathbb{B}+L_{1}\left\|w_{1, a}^{i}-w_{1, b}^{i}\right\| \mathbb{B}+L_{2}\left\|\prod_{j \in I}\left\{h^{j}\left(x_{a}^{j}\right)\right\}-\prod_{j \in I}\left\{h^{j}\left(x_{b}^{j}\right)\right\}\right\| \mathbb{B}\right) \\
& \subseteq \prod_{i \in I}\left(F^{i}\left(x_{b}^{i}, w_{1, b}^{i}, w_{2, b}^{i}\right)+L\left\|x_{a}^{i}-x_{b}^{i}\right\| \mathbb{B}+L_{1}\left\|w_{1, a}^{i}-w_{1, b}^{i}\right\| \mathbb{B}+L_{2} l\left\|x_{a}-x_{b}\right\| \mathbb{B}\right) \\
& \subseteq \prod_{i \in I}\left(F^{i}\left(x_{b}^{i}, w_{1, b}^{i}, w_{2, b}^{i}\right)+L\left\|x_{a}-x_{b}\right\| \mathbb{B}+L_{1}\left\|w_{1, a}-w_{1, b}\right\| \mathbb{B}+L_{2} l\left\|x_{a}-x_{b}\right\| \mathbb{B}\right) \\
& \subseteq \prod_{i \in I}\left(F^{i}\left(x_{b}^{i}, w_{1, b}^{i}, w_{2, b}^{i}\right)+\left(L+L_{2} l\right)\left\|x_{a}-x_{b}\right\| \mathbb{B}+L_{1}\left\|w_{1, a}-w_{1, b}\right\| \mathbb{B}\right) \\
& \subseteq \prod_{i \in I}\left(F^{i}\left(x_{b}^{i}, w_{1, b}^{i}, w_{2, b}^{i}\right)\right)+\left(L+L_{2} l\right)\left\|x_{a}-x_{b}\right\| \mathbb{B}+L_{1}\left\|w_{1, a}-w_{1, b}\right\| \mathbb{B} \\
& =F\left(x_{b}, w_{1, b}\right)+\left(L+L_{2} l\right)\left\|x_{a}-x_{b}\right\| \mathbb{B}+L_{1}\left\|w_{1, a}-w_{1, b}\right\| \mathbb{B}
\end{aligned}
$$

Henzinger, Kim G Larsen, et al. Contracts for system design. Foundations and Trends@ in Electronic Design Automation, 12(2-3):124-400, 2018.

[7] Yuxiao Chen, James Anderson, Karan Kalsi, Steven H Low, and Aaron D Ames. Compositional set invariance in network systems with assume-guarantee contracts. In 2019 American Control Conference (ACC), pages 1027-1034. IEEE, 2019.

[8] C. Conte, N.R. Voellmy, M.N. Zeilinger, M. Morari, and C.N. Jones. Distributed synthesis and control of constrained linear systems. In American Control Conference, pages 6017-6022, 2012.

[9] S. Coogan and M. Arcak. A dissipativity approach to safety verification for interconnected systems. IEEE Transactions on Automatic Control, 60(6):1722-1727, 2015.

[10] E. Dallal and P. Tabuada. On compositional symbolic controller synthesis inspired by small-gain theorems. In IEEE Conference on Decision and Control, pages 6133-6138, 2015.

[11] S. Dashkovskiy, B.S. Rüffer, and F.R. Wirth. An iss small gain theorem for general networks. Mathematics of Control, Signals, and Systems, 19(2):93-122, 2007.

[12] C.A. Desoer and M. Vidyasagar. Feedback systems: inputoutput properties, volume 55. Siam, 1975.

[13] A. El-Guindy, Y.C. Chen, and M. Althoff. Compositional transient stability analysis of power systems via the computation of reachable sets. In American Control Conference, pages 2536-2543. IEEE, 2017.

[14] Alina Eqtami and Antoine Girard. A quantitative approach on assume-guarantee contracts for safety of interconnected systems. In 2019 18th European Control Conference (ECC), pages 536-541. IEEE, 2019.
[15] Goran Fedja Frehse. Compositional verification of hybrid systems using simulation relations. [Sl: sn], 2005.

[16] Kasra Ghasemi, Sadra Sadraddini, and Calin Belta. Compositional synthesis via a convex parameterization of assume-guarantee contracts. In Proceedings of the 23rd International Conference on Hybrid Systems: Computation and Control, pages 1-10, 2020.

[17] R. Goebel, R. G. Sanfelice, and A. R. Teel. Hybrid Dynamical Systems: Modeling, Stability, and Robustness. Princeton University Press, New Jersey, 2012.

[18] T.A. Henzinger, M. Minea, and V. Prabhu. Assumeguarantee reasoning for hierarchical hybrid systems. In International Workshop on Hybrid Systems: Computation and Control, pages 275-290. Springer, 2001.

[19] Z-P Jiang, Andrew R Teel, and L Praly. Small-gain theorem for iss systems and applications. Mathematics of Control, Signals and Systems, 7(2):95-120, 1994.

[20] F. Kerber and AJ. Van Der Schaft. Assume-guarantee reasoning for linear dynamical systems. In European Control Conference, pages 5015-5020. IEEE, 2009.

[21] Hassan K Khalil. Noninear systems. Prentice-Hall, New Jersey, 2(5):5-1, 1996.

[22] E.S. Kim, M. Arcak, and S.A. Seshia. Compositional controller synthesis for vehicular traffic networks. In IEEE Conference on Decision and Control, pages 6165-6171, 2015.

[23] E.S. Kim, M. Arcak, and S.A. Seshia. A small gain theorem for parametric assume-guarantee contracts. In Proceedings of the 20th International Conference on Hybrid Systems: Computation and Control, pages 207-216. ACM, 2017.

[24] A. Le Coënt, L. Fribourg, N. Markey, F. De Vuyst, and L. Chamoin. Distributed synthesis of state-dependent 
switching control. In International Workshop on Reachability Problems, pages 119-133, 2016.

[25] E.A. Lee and S.A. Seshia. Introduction to embedded systems: A cyber-physical systems approach. Mit Press, 2016.

[26] Kaushik Mallik, Anne-Kathrin Schmuck, Sadegh Soudjani, and Rupak Majumdar. Compositional synthesis of finitestate abstractions. IEEE Transactions on Automatic Control, 64(6):2629-2636, 2018.

[27] P.-J. Meyer and D.V. Dimarogonas. Compositional abstraction refinement for control synthesis. Nonlinear Analysis: Hybrid Systems, 27:437-451, 2018.

[28] Pierre-Jean Meyer, Antoine Girard, and Emmanuel Witrant. Compositional abstraction and safety synthesis using overlapping symbolic models. IEEE Transactions on Automatic Control, 63(6):1835-1841, 2017.

[29] P. Nilsson and N. Ozay. Synthesis of separable controlled invariant sets for modular local control design. In American Control Conference, pages 5656-5663, 2016.

[30] S.V. Raković, B. Kern, and R. Findeisen. Practical set invariance for decentralized discrete time systems. In IEEE Conference on Decision and Control, pages 3283-3288, 2010.

[31] R Tyrrell Rockafellar and Roger J-B Wets. Variational analysis. Springer Science Business Media, 2009.

[32] A. Saoud, A. Girard, and L. Fribourg. Contract based design of symbolic controllers for interconnected multiperiodic sampled-data systems. In Conference on Decision and Control, pages 773-779, 2018.

[33] A. Saoud, A. Girard, and L. Fribourg. Contract based design of symbolic controllers for vehicle platooning. In Proceedings of the 21st International Conference on Hybrid Systems: Computation and Control, HSCC '18, pages 277-278, 2018.

[34] A. Saoud, A. Girard, and L. Fribourg. On the composition of discrete and continuous-time assume-guarantee contracts for invariance. In European Control Conference, pages 435-440, 2018.

[35] A. Saoud, P Jagtap, M Zamani, and A. Girard. Compositional abstraction-based synthesis for cascade discrete-time control systems. IFAC-PapersOnLine, 51(16):13 - 18, 2018. 6th IFAC Conference on Analysis and Design of Hybrid Systems.

[36] Adnane Saoud. Compositional and Efficient Controller Synthesis for Cyber-Physical Systems. PhD thesis, Universié Paris Saclay, 2019.

[37] C. Sloth, G.J. Pappas, and R. Wisniewski. Compositional safety analysis using barrier certificates. In International Conference on Hybrid Systems: Computation and Control, pages 15-24, 2012.

[38] S. W Smith, P. Nilsson, and N. Ozay. Interdependence quantification for compositional control synthesis with an application in vehicle safety systems. In Conference on Decision and Control, pages 5700-5707. IEEE, 2016.

[39] AJ Van der Schaft. Equivalence of dynamical systems by bisimulation. IEEE transactions on automatic control, 49(12):2160-2172, 2004.

[40] Majid Zamani and Murat Arcak. Compositional abstraction for networks of control systems: A dissipativity approach. IEEE Transactions on Control of Network Systems, 5(3):1003-1015, 2017.

[41] Daniele Zonetti, Adnane Saoud, Antoine Girard, and Laurent Fribourg. A symbolic approach to voltage stability and power sharing in time-varying dc microgrids. In 2019 18th European Control Conference (ECC), pages 903-909. IEEE, 2019. 